\title{
Pacific
}

Journal of

Mathematics

\section{THE CORESTRICTION OF VALUED DIVISION ALGEBRAS OVER HENSELIAN FIELDS. I}

YoOn SUNG HWANG 


\title{
THE CORESTRICTION OF VALUED DIVISION ALGEBRAS OVER HENSELIAN FIELDS I
}

\author{
YOON SUNG HWANG
}

\begin{abstract}
When $L / F$ is an unramified extension of Henselian fields, we analyze the underlying division algebra ${ }^{c} D$ of the corestriction $\operatorname{cor}_{L / F}(D)$ of a tame division algebra $D$ over $L$ with respect to the unique valuations on ${ }^{c} D$ and $D$ extending the valuations on $F$ and $L$. We show that the value group of ${ }^{c} D$ lies in the value group of $D$ and for the center of residue division algebra, $Z(\bar{c} \bar{D}) \subseteq \mathcal{N}(Z(\bar{D}) / \bar{F})^{1 / k}$, where $\mathcal{N}(Z(\bar{D}) / \bar{F})$ is the normal closure of $Z(\bar{D})$ over $\bar{F}$ and $k$ is an integer depending on which roots of unity lie in $F$ and $L$.
\end{abstract}

\section{Introduction.}

For any finite separable extension $L / F$ of fields and any central simple algebra $A$ over $L$, the corestriction of $A$ is a central simple $F$-algebra obtained as the fixed point algebra under a Galois group action (cf. [Ri]). This induces the map from the Brauer group $\mathrm{Br}(L)$ to $\mathrm{Br}(F)$ corresponding to the homological corestriction. Though this algebraic corestriction is an important tool in the theory of division algebras, it is actually very hard to work with. To gain a better insight into the behavior of the corestriction, we analyze here the corestriction for valued division algebras over Henselian valued fields, for which there is a well-developed structure theory. We will here concentrate on the case when $L$ is inertial (unramified) over $F$. In a subsequent paper [H2], we will consider the more general case when $L$ is tame over $F$, i.e. $\operatorname{char}(\bar{F}) \nmid[L: F]$, where $\bar{F}$ is the residue field of the valuation on $F$.

For any ring $R$ we write $Z(R)$ and $R^{*}$ for the center of $R$ and the group of units of $R$, respectively. We will consider only central simple algebras $A$ finite-dimensional over a field $F$. By Wedderburn's theorem, $A \cong M_{n}(D)$, a matrix ring over a division algebra $D$, which is called the underlying division algebra of $A$.

A valued field $(F, v)$ is called Henselian if $v$ extends uniquely to each field algebraic over $F$. For a nice account for several other characterizations of Henselian valuations, see Ribenboim's paper [Rb]. Recall (e.g. from [W1]) that if $D$ is a central division algebra over a Henselian valued field $(F, v)$, there exists one and only one valuation on $D$ extending $v$ on $F$. 
Let $(L, v) \supseteq(F, v)$ be a finite separable inertial extension of Henselian fields. This means that $[\bar{L}: \bar{F}]=[L: F]$ and $\bar{L}$ is separable over $\bar{F}$. Let $D$ be a central division algebra over $L$. We denote by ${ }^{c} D$ the underlying division algebra of the corestriction $\operatorname{cor}_{L / F}(D)$ of $D$. If $D$ is inertially split over $L$, i.e., split by the maximal unramified extension of $L$, we have good information obtained homologically using [JW2, Sec. 5]. Also, we can analyze a tame and totally ramified division algebra since it is isomorphic to a tensor product of symbol algebras (cf. Prop. 1.6). Furthermore, since a tame division algebra over $L$ decomposes in the Brauer group to $S \otimes_{L} T$ with $S$ inertially split and $T$ tame and totally ramified (cf. Prop. 1.7), we obtain information about a tame division algebra by combining the results about $S$ and $T$. Our basic results are summarized in the following table. Here $\Gamma_{D}$ is the value group of the valuation on $D$ and $\bar{D}$ is the residue division ring of the valuation ring of $D$. Also $\mathcal{N}(Z(\bar{D}) / \bar{F})$ denotes the normal closure of $Z(\bar{D})$ over $\bar{F}, N_{\bar{L} / \bar{F}}$ denotes the norm map from $\bar{L}$ to $\bar{F}$, and $\theta_{D}$ is the map of (1.5) below, so $\operatorname{ker}\left(\theta_{D}\right)$ is a subgroup of $\Gamma_{D} / \Gamma_{L}$.

\begin{tabular}{|c|c|c|}
\hline$D$ & $\Gamma_{{ } D}$ & $Z\left(\overline{{ }^{c} D}\right)$ \\
\hline $\begin{array}{c}\text { inertially split } \\
\text { (Th. 2.4) }\end{array}$ & $\Gamma_{{ } D} \subseteq \Gamma_{D}$ & $Z\left(\overline{{ }^{c} D}\right) \subseteq \mathcal{N}(Z(\bar{D}) / \bar{F})$ \\
\hline $\begin{array}{c}\text { tame, totally } \\
\text { ramified } \\
\text { (Prop. 3.2, Th. 3.9) }\end{array}$ & $\Gamma_{{ } D} \subseteq \Gamma_{D}$ & $\begin{array}{c}Z\left(\overline{{ }^{c} D}\right) \subseteq \bar{F}\left(N_{\bar{L} / \bar{F}}(\bar{L})^{1 / t}\right) \\
t=\exp \left(\Gamma_{D} / \Gamma_{L}\right) \\
\left(\text { if } \mu_{t} \subseteq F\right)\end{array}$ \\
\hline $\begin{array}{c}\text { tame } \\
\text { (Th. 4.5, 4.6) }\end{array}$ & $\Gamma_{{ } D} \subseteq \Gamma_{D}$ & $\begin{array}{c}Z\left(\overline{{ }^{c} D}\right) \subseteq \mathcal{N}(Z(\bar{D}) / \bar{F})^{1 / k} \\
k \mid \exp \left(\operatorname{ker} \theta_{D}\right)\end{array}$ \\
\hline
\end{tabular}

The integer $k$ in the table above depends not only on $\Gamma_{D} / \Gamma_{L}$ and $[L: F]$ but also on which roots of unity lie in $F$. One of the interesting results of the investigation is to see how heavily the corestriction depends on the roots of unity in $F$ and $L$.

Here is an overview of the paper: After giving some preliminary results in Section 1, we will analyze the corestriction of inertially split division algebras in Section 2. In Section 3, we will consider the corestriction of tame and totally ramified division algebras $T$ over $L$ (i.e. $\left|\Gamma_{T}: \Gamma_{L}\right|=[T: L]$ and $\operatorname{char}(\bar{L}) \nmid[T: L]$ ) when $\mu_{t} \subseteq F$ with $t=\exp \left(\Gamma_{T} / \Gamma_{L}\right)$ (i.e. $F$ has a $t$-th primitive root of unity). Finally, in Section 4 we will analyze the corestriction of tame division algebras. (In the Appendix, we will give an explicit formula of the homological corestriction.) 
This paper constitutes part of the author's doctoral dissertation. The author thanks his thesis advisor Adrian Wadsworth for his great and kind advice.

\section{Preliminaries.}

Let $F \subseteq K$ be fields with $K$ algebraic over $F$. We write $\operatorname{Gal}(K / F)$ for the profinite group of all $F$-automorphisms of $K$. If $H$ is any subset of $\operatorname{Gal}(K / F)$, we write $\mathcal{F}(H)$ for the fixed field of $H$. We say $K$ is normal over $F$ if $K$ is the splitting field of some family of polynomials over $F ; K$ is Galois over $F$ if $K$ is both normal and separable (if and only if $F=\mathcal{F}(\operatorname{Gal}(K / F))$ ). We write $\mathcal{N}(K / F)$ for the normal closure of $K$ over $F$. Also, if $[K: F]<\infty$, $N_{K / F}$ denotes the norm map from $K$ to $F$. We write $F_{\text {sep }}$ for the separable closure of $F$.

For a central simple $F$-algebra $A,[A]$ denotes the class of $A$ in the Brauer group $\operatorname{Br}(F)$ of $F$. If $B$ is another central simple $F$-algebra with $[B]=[A]$, we write $A \sim B$. For any field $K \supseteq F$, let $\mathrm{Br}(K / F)$ denote the relative Brauer group of $K / F$, which is the kernel of the canonical "restriction" homomorphism $\mathrm{Br}(F) \rightarrow \mathrm{Br}(K)$ given by $[A] \rightarrow\left[A \otimes_{F} K\right]$.

Here is our notation for certain central simple $F$-algebras: $(K / F, G, f)$ is the crossed product algebra determined by a 2-cocycle $f: G \times G \rightarrow K^{*}$, where $K$ is a finite dimensional Galois extension field of $F$ with Galois group $G$;

$(K / F, \sigma, a)_{n}$ is the cyclic $F$-algebra generated over $K$ by a single element $x$ with defining relations $x c x^{-1}=\sigma(c)$ for all $c \in K$ and $x^{n}=a \in F^{*}$, where $K$ is a Galois extension of $F$ with cyclic Galois group generated by $\sigma$ and $n=[K: F]$;

$(a, b ; F)_{n}$ is the symbol algebra generated over $F$ by $i$ and $j$ with defining relations: $i^{n}=a, j^{n}=b, i j=\omega j i$, where $\omega \in F^{*}$ is a primitive $n$-th root of unity and $a, b \in F^{*}$. Since a symbol algebra depends on a choice of a primitive $n$-th root of unity $\omega$, we will sometimes write $(a, b, \omega ; F)_{n}$ for $(a, b ; F)_{n}$. In writing symbol algebra $(a, b ; F)_{n}$ there is an implicit choice made of which primitive $n$-th root of unity $\omega$ to use. When this has been done, then for an integer $n^{\prime}$ dividing $n,(a, b ; F)_{n^{\prime}}$ denotes the symbol algebra built using the primitive $n^{\prime}$-th root of unity $\omega^{n / n^{\prime}}$.

Recall that the cyclic algebra $(K / F, \sigma, a)_{n}$ corresponds to the cohomology class of the cup product

$$
\left[\delta\left(\inf _{G_{0}}^{G}(f)\right)\right] \cup(a)
$$

in the continuous cohomology group $H_{c}^{2}\left(G, F_{\text {sep }}^{*}\right)$,

where $G=\operatorname{Gal}\left(F_{\text {sep }} / F\right)$ is the absolute Galois group of $F, G_{0}=\operatorname{Gal}(K / F)$ 
$=\langle\sigma\rangle, f \in H^{1}\left(G_{0}, \mathbb{Q} / \mathbb{Z}\right)=\operatorname{Hom}\left(G_{0}, \mathbb{Q} / \mathbb{Z}\right)$ is defined by $f(\sigma)=\frac{1}{n}+\mathbb{Z}$, $\delta: H^{1}(G, \mathbb{Q} / \mathbb{Z}) \rightarrow H^{2}(G, \mathbb{Q} / \mathbb{Z})$ is the connecting homomorphism, and $a \in$ $F^{*}=H^{0}\left(G, F_{\text {sep }}^{*}\right)$.

In fact, the corestriction (or transfer) homomorphism arose from the cohomology of groups, and the algebraic corestriction is compatible with the homological corestriction via the crossed product construction. Let $G$ be a group and let $A$ be a left $G$ module. Let $H$ be a subgroup of $G$ of finite index $n$. For $m \geq 0$ an integer, $H^{m}(G, A)$ (resp. $\left.H^{m}(H, A)\right)$ denotes the $m$-th cohomology group of $G$ (resp. $H$ ) with coefficients in $A$.

For each $m$, there is the corestriction (or transfer) $\operatorname{cor}_{H}^{G}: H^{m}(H, A) \rightarrow$ $H^{m}(G, A)$. (See [B, III, Sec. 9].) We give an explicit formula describing this homological corestriction for $m \geq 0$. Let $\mathcal{R}=\left\{\rho_{1}, \ldots, \rho_{n}\right\}$ be a set of representatives of the left cosets of $H$ in $G$. For $m=0, H^{0}(H, A)=A^{H}:=$ $\{a \in A \mid \sigma a=a$ for all $\sigma \in H\}$ and $H^{0}(G, A)=A^{G}$, and $\operatorname{cor}_{H}^{G}: H^{0}(H, A) \rightarrow$ $H^{0}(G, A)$ is given by $a \mapsto \sum_{i=1}^{n} \rho_{i} a$ for $a \in H^{0}(H, A)=A^{H}$. Given $\rho_{i} \in \mathcal{R}$ and $\sigma \in G$, there are uniquely determined elements $\rho_{\sigma_{*}(i)} \in \mathcal{R}$ and $\delta\left(\sigma, \rho_{i}\right) \in H$ such that

$$
\sigma \rho_{i}=\rho_{\sigma_{*}(i)} \delta\left(\sigma, \rho_{i}\right),
$$

where the map $\sigma \mapsto \sigma_{*}$ is a homomorphism from $G$ to the symmetric group $S_{n}$. Now take any $f \in Z^{m}(H, A)$, the group of $m$ cocycles of $H$ with values in $A$; define a function $\operatorname{cor}_{H}^{G}(f) \in Z^{m}(G, A)$ as follows: for $\sigma_{1}, \ldots, \sigma_{m} \in G$

$$
\begin{aligned}
& \operatorname{cor}_{H}^{G}(f)\left(\sigma_{1}, \ldots, \sigma_{m}\right) \\
&=\sum_{i=1}^{n} \rho_{\left(\sigma_{1} \cdots \sigma_{m}\right)_{*}(i)}\left[f \left(\delta\left(\sigma_{1}, \rho_{\left(\sigma_{2} \cdots \sigma_{m}\right)_{*}(i)}\right),\right.\right. \\
&\left.\left.\ldots, \delta\left(\sigma_{j}, \rho_{\left(\sigma_{j+1} \cdots \sigma_{m}\right)_{*}(i)}\right), \ldots, \delta\left(\sigma_{m}, \rho_{i}\right)\right)\right] .
\end{aligned}
$$

(We will deduce this formula from [Ta, p. 259] in the Appendix.) For $m \geq 1$, the homological corestriction $\operatorname{cor}_{H}^{G}: H^{m}(H, A) \rightarrow H^{m}(G, A)$ is induced by the map $f \mapsto \operatorname{cor}_{H}^{G}(f)$ given in (1.3) above. Recall that the (homological) corestriction map commutes with inflation and connecting homomorphisms (cf. [We, Prop 2.4.5 and Prop. 2.4.7]).

Recall the following case of the projection formula which will be used often in this paper (cf. [Ti, Th. 3.1]).

Proposition 1.4 (Projection Formula). Let $L / F$ be finite dimensional and separable. Assume $F$ contains a primitive $n$-th root of unity. If $\alpha \in L^{*}$ and $b \in F^{*}$, then $\operatorname{cor}_{L / F}\left((\alpha, b ; L)_{n}\right) \sim\left(N_{L / F}(\alpha), b ; F\right)_{n}$ in $\operatorname{Br}(F)$.

The valued division algebra $(D, v)$ is a division ring $D$ with valuation $v$, that is, a function $v: D^{*} \rightarrow \Gamma$ (a totally ordered abelian group written 
additively) such that for all $a, b$ in $D^{*}, v(a b)=v(a)+v(b)$ and $v(a+$ $b) \geq \min (v(a), v(b))$ if $a \neq-b$. Associated to $v$, we have its value group $\Gamma_{D}=v\left(D^{*}\right)$; the valuation ring $V_{D}=\left\{d \in D^{*} \mid v(d) \geq 0\right\} \cup\{0\}$; the unique maximal left ideal $M_{D}$ of $V_{D}, M_{D}=\left\{d \in D^{*} \mid v(d)>0\right\} \cup\{0\}$; the group of $v$-units of $D^{*}, U_{D}=V_{D}-M_{D}=V_{D}^{*}$; the residue division ring $\bar{D}=V_{D} / M_{D}$. If $F$ is the center $Z(D)$ of $D$, there is a well-defined epimorphism

$$
\theta_{D}: \Gamma_{D} / \Gamma_{F} \rightarrow \operatorname{Gal}(Z(\bar{D}) / \bar{F})
$$

induced by $\alpha: D^{*} \rightarrow \operatorname{Gal}(Z(\bar{D}) / \bar{F})$ which is given by $d \mapsto \overline{c_{d}}$ where $\overline{c_{d}}$ is the map induced by conjugation by $d$ (cf. $[\mathbf{J W 2}, 1.6]$ ).

Let $(F, v)$ be a Henselian valued field. Let $D$ be a central division $F$ algebra (with a unique valuation extending $v$ on $F$ ). We say $D$ is tame and totally ramified over $F$ if $\operatorname{char}(\bar{F}) \nmid[D: F]$ and $\left|\Gamma_{D}: \Gamma_{F}\right|=[D: F] . D$ is said to be inertially split over $F$ if $D$ is split by $F_{n r}$ where $F_{n r}$ is the maximal unramified extension in some algebraic closure of $F$. Also, $D$ is said to be tame if $\operatorname{char}(\bar{F})=0$ or $\operatorname{char}(\bar{F})=q \neq 0$ and the $q$-primary component of $D$ is split by $F_{n r}$. (See [JW2, Lemma 5.1] and [JW2, Lemma 6.1] for other characterizations of inertially split and tame division algebras.) Recall also that $D$ is said to be inertial over $F$ if $[\bar{D}: \bar{F}]=[D: F]$ and $Z(\bar{D})=\bar{F}$. Let

$$
\begin{aligned}
\mathcal{D}(F) & =\{D \mid D \text { is a central division F-algebra with }[D: F]<\infty\} \\
\mathcal{D}_{t t r} & =\{D \in \mathcal{D}(F) \mid D \text { is tame and totally ramified over } F\} \\
\mathcal{D}_{i}(F) & =\{D \in \mathcal{D}(F) \mid D \text { is inertial over } F\} \\
\mathcal{D}_{i s}(F) & =\{D \in \mathcal{D}(F) \mid D \text { is inertially split over } F\} \text { and } \\
\mathcal{D}_{t}(F) & =\{D \in \mathcal{D}(F) \mid D \text { is tame over } F\} .
\end{aligned}
$$

It is clear that $\mathcal{D}_{i}(F) \subseteq \mathcal{D}_{i s}(F) \subseteq \mathcal{D}_{t}(F)$ and $\mathcal{D}_{t t r}(F) \subseteq \mathcal{D}_{t}(F)$.

Given $(D, v)$ a valued central division $F$-algebra, one has the "fundamental inequality" $[D: F] \geq[\bar{D}: \bar{F}] \cdot\left|\Gamma_{D}: \Gamma_{F}\right|$ (cf. [S, p. 21]). We say $D$ is defectless over $F$ if equality holds.

Throughout this paper, we will assume that $L / F$ is a finite separable inertial (unramified) extension of Henselian valued fields. We now recall some facts about inertial extensions as summarized in [JW2, Sec. 1]. Let $F_{n r}$ denote the maximal unramified extension of $F$ in some algebraic closure $F_{a l g}$ of $F$. Recall that $\Gamma_{F_{n r}}=\Gamma_{F}, \overline{F_{n r}}$ is the separable closure $\bar{F}_{\text {sep }}$ of $\bar{F}$, and $F_{n r}$ is Galois over $F$. Also, for any field $E$ with $F \subseteq E \subseteq F_{a l g}$ and $[E: F]<\infty, E$ is inertial over $F$ if and only if $E \subseteq F_{n r}$. Further, the map $L \mapsto \bar{L}$ gives a $1-1$ correspondence between fields $L$ with $F \subseteq L \subseteq F_{n r}$ and fields $\widetilde{L}$ with $\bar{F} \subseteq \widetilde{L} \subseteq \overline{F_{n r}}$. Given a field $\widetilde{L}$ with $\bar{F} \subseteq \widetilde{L} \subseteq \bar{F}_{\text {sep }}$ and 
$[\widetilde{L}: \bar{F}]<\infty$, the corresponding $L$ (inertial over $F$ with $\bar{L}=\widetilde{L}$ ) will be called the inertial lift of $\widetilde{L}$ over $F$.

We now finish this section by recalling two propositions which will be very useful for this paper. See [D2, Th. 1] and [JW2, Lemma 6.2, Th. 6.3] for proofs of Props. 1.6 and 1.7, respectively.

Proposition 1.6 (Draxl's decomposition theorem). If $D \in \mathcal{D}(F)$ is tame and totally ramified over a Henselian field $F, D$ is isomorphic to a tensor product of symbol algebras.

Proposition 1.7. If $D \in \mathcal{D}(F)$ is tame over a Henselian field $F$, then there exist $S \in \mathcal{D}_{\text {is }}(F)$ and $T \in \mathcal{D}_{\text {ttr }}(F)$ such that $D \sim S \otimes_{F} T$ in $\operatorname{Br}(F)$. (Such $S$ and $T$ are not unique.) Furthermore, if $D \sim S \otimes_{F} T$ is such a decomposition,

$$
\begin{aligned}
Z(\bar{D}) & =\mathcal{F}\left(\theta_{S}\left(\left(\Gamma_{S} \cap \Gamma_{T}\right) / \Gamma_{F}\right)\right) \subseteq Z(\bar{S}), \\
\Gamma_{D} & =\Gamma_{S}+\Gamma_{T} \text { and } \operatorname{ker}\left(\theta_{D}\right)=\Gamma_{T} / \Gamma_{F} .
\end{aligned}
$$

\section{Inertially split division algebras.}

If $D \in \mathcal{D}_{i s(F)}$, we have good information about $D$ homologically, which is obtained in [JW2, Sec. 5]. We will give a brief summary on this. (See [JW2, Th. 5.6]).

We fix a Henselian valued field $(F, v)$. Let $\Delta$ be the divisible hull of $\Gamma_{F}$, so $\Delta \simeq \Gamma_{F} \otimes_{\mathbb{Z}} \mathbb{Q}$. Let $G=\operatorname{Gal}\left(F_{n r} / F\right) \cong \operatorname{Gal}\left(\bar{F}_{\text {sep }} / \bar{F}\right)$, a profinite group. Let $U=U_{F_{n r}}$ the group of $v$-units of $F_{n r}$. Since $\Gamma_{F_{n r}}=\Gamma_{F}$ (on which $G$ acts trivially), we have a short exact sequence of discrete $G$-modules:

$$
1 \rightarrow U \rightarrow F_{n r}^{*} \stackrel{v}{\rightarrow} \Gamma_{F} \rightarrow 0
$$

From this short exact sequence, we have the following exact sequence, which will be the basic focus of our attention:

$$
0 \rightarrow H_{c}^{2}(G, U) \rightarrow H_{c}^{2}\left(G, F_{n r}^{*}\right) \stackrel{\gamma}{\rightarrow} \operatorname{Hom}_{c}\left(G, \Delta / \Gamma_{F}\right)
$$

where $H_{c}^{2}$ and $\mathrm{Hom}_{c}$ denote the second (continuous) cohomology group and the continuous homomorphism group, respectively.

In this exact sequence, $H_{c}^{2}\left(G, F_{n r}^{*}\right) \cong B r\left(F_{n r} / F\right)$ and $H_{c}^{2}(G, U) \cong I B r(F)$, where $\operatorname{IBr}(F)=\left\{[D] \in \operatorname{Br}(F) \mid D \in D_{i}(F)\right.$ i.e., $D$ is inertial over $\left.F\right\}$. (See [JW2, Th. 5.6].) Also, $\operatorname{IBr}(F)$ is isomorphic to $B r(\bar{F})$ via the isomorphism $\beta$ given by $[D] \rightarrow[\bar{D}]$. (See [JW2, Th. 2.8].) Further, if $\alpha: H_{c}^{2}(G, U) \rightarrow$ $H_{c}^{2}\left(G,{\overline{F_{n r}}}^{*}\right)=H_{c}^{2}\left(G, \bar{F}_{s e p}^{*}\right)$ is the map induced by a map $U \rightarrow \bar{F}_{\text {sep }}^{*}$ given 
by $u \mapsto \bar{u}$, the following diagram commutes: (See [JW2, Examples 2.4, Prop. 2.5].)

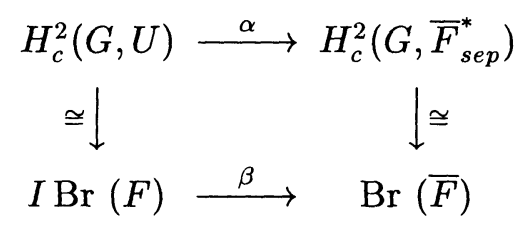

where the vertical isomorphisms are given by crossed product construction. We now identify $H_{c}^{2}\left(G, F_{n r}^{*}\right)$ with $\operatorname{Br}\left(F_{n r} / F\right)$. For any $D \in \mathcal{D}_{i s}(F)$, as $[D] \in \operatorname{Br}\left(F_{n r} / F\right)$, set $h_{D}=\gamma[D] \in \operatorname{Hom}_{c}\left(G, \Delta / \Gamma_{F}\right)$, where $\gamma$ is the map in (2.1); further, let $\widetilde{h}_{D}: G / \operatorname{ker}\left(h_{D}\right) \rightarrow \operatorname{im}\left(h_{D}\right)$ be the isomorphism induced by $h_{D}$. Then
$\left\{\begin{array}{l}(1) \\ (2)\end{array}\right.$
$\operatorname{im}\left(h_{D}\right)=\Gamma_{D} / \Gamma_{F}$
and $\mathcal{F}\left(\operatorname{ker}\left(h_{D}\right)\right)$
is the inertial lift of $Z(\bar{D})$
(2) (After identifying $G / \operatorname{ker}\left(h_{D}\right)$ with Gal $\left.(Z(\bar{D}) / \bar{F})\right) \tilde{h}_{D}=\theta_{D}^{-1}$,

where $\theta_{D}: \Gamma_{D} / \Gamma_{F} \rightarrow$ Gal $(Z(\bar{D}) / \bar{F})$ is the map of (1.5) induced by conjugation. See [JW2, Th. 5.6] for the proof.

Now let $(L, v) \supseteq(F, v)$ be a finite (separable) inertial extension of Henselian fields. Since $L$ is inertial over $F, L \subseteq F_{n r}$ so $L_{n r}=F_{n r}$. Let

$$
\begin{aligned}
& G=\operatorname{Gal}\left(F_{n r} / F\right) \cong \operatorname{Gal}\left(\bar{F}_{s e p} / \bar{F}\right) \text { and } \\
& H=\operatorname{Gal}\left(F_{n r} / L\right)=\operatorname{Gal}\left(L_{n r} / L\right) \cong \operatorname{Gal}\left(\bar{L}_{s e p} / \bar{L}\right)
\end{aligned}
$$

Then $H$ is a subgroup of finite index $|G: H|=[L: F]=[\bar{L}: \bar{F}]$.

Note that, for $i \geq 0$ the corestriction map $\operatorname{cor}_{H}^{G}$ from $H^{i}\left(H, F_{n r}^{*}\right)$ to $H^{i}\left(G, F_{n r}^{*}\right)$ sends the continuous cohomology group $H_{c}^{i}\left(H, F_{n r}^{*}\right)$ into the continuous cohomology group $H_{c}^{i}\left(G, F_{n r}^{*}\right)$.

Theorem 2.4. Let $(L, v) \supseteq(F, v)$ be a finite inertial extension of Henselian fields. Suppose $D \in \mathcal{D}_{\text {is }}(L)$. Then,

(a) ${ }^{c} D \in \mathcal{D}_{i s}(F)$

(b) $\Gamma_{{ } D} \subseteq \Gamma_{D}$

(c) $Z\left(\overline{{ }^{c} D}\right) \subseteq \mathcal{N}(Z(\bar{D}) / \bar{F})$, the normal closure of $Z(\bar{D})$ over $\bar{F}$.

(d) If $D \in \mathcal{D}_{i}(L)$, then ${ }^{c} D \in \mathcal{D}_{i}(F)$ and ${ }^{\bar{c} D} \sim \operatorname{cor}_{\bar{L} / \bar{F}}(\bar{D})$ in $\operatorname{Br}(\bar{F})$. 
(So, if $D \in \mathcal{D}_{t}(L)$, then ${ }^{c} D \in \mathcal{D}_{t}(F)$.)

Proof. (a) Let $N=\mathcal{N}(L / F)$. As $F_{n r}$ is normal over $F, N \subseteq F_{n r}$, so $N_{n r}=$ $F_{n r}$. Let $\mathcal{R}$ be a set of representatives of the left cosets of $\operatorname{Gal}(N / L)$ in $\operatorname{Gal}(N / F)$. Then, by [D2, p. 52, Cor. 1], ${ }^{c} D \otimes_{F} N \sim \operatorname{cor}_{L / F}(D) \otimes_{F} N \cong$ $\otimes_{\rho \in \mathcal{R}}^{\rho}\left(D \otimes_{L} N\right) \in \operatorname{Br}\left(N_{n r} / N\right)$ since $D \in \mathcal{D}_{i s}(L)$, where ${ }^{\rho}\left(D \otimes_{L} N\right)$ means $D \otimes_{L} N$ made into an $N$-algebra by $(d \otimes n) \cdot n^{\prime}=d \otimes n \rho^{-1}\left(n^{\prime}\right)$. So, ${ }^{c} D$ is split by $N_{n r}=F_{n r}$, hence ${ }^{c} D \in \mathcal{D}_{i s}(F)$.

From this and definition of tame division algebras, it is clear that if $D \in$ $\mathcal{D}_{t}(L)$, then ${ }^{c} D \in \mathcal{D}_{t}(F)$.

(b) Let $H=\operatorname{Gal}\left(L_{n r} / L\right)=\operatorname{Gal}\left(F_{n r} / L\right), G=\operatorname{Gal}\left(F_{n r} / F\right), \Delta=$ the divisible hull of $\Gamma_{F}=\Gamma_{L}$, and $U=U_{F_{n r}}=U_{L_{n r}}$. Then since the corestriction map is functorial and commutes with the connecting homomorphism, the following diagram is commutative,

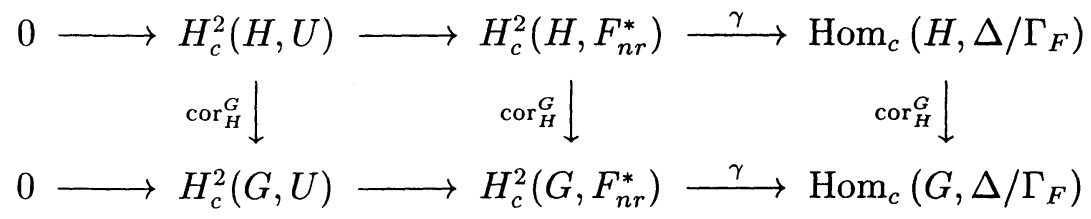

where the row exact sequences are those of (2.1). As done in (2.3), we identify $H_{c}^{2}\left(H, F_{n r}^{*}\right)$ and $H_{c}^{2}\left(G, F_{n r}^{*}\right)$ with $\operatorname{Br}\left(L_{n r} / L\right)$ and $\operatorname{Br}\left(F_{n r} / F\right)$, respectively. As $D \in \mathcal{D}_{i s}(L)$ and by (a), ${ }^{c} D \in \mathcal{D}_{i s}(F)$. Set $h_{D}=\gamma[D] \in$ $\operatorname{Hom}_{c}\left(H, \Delta / \Gamma_{F}\right)$ and $h_{c_{D}}=\gamma\left[{ }^{c} D\right] \in \operatorname{Hom}_{c}\left(G, \Delta / \Gamma_{F}\right)$. Since the diagram above is commutative, $h_{c_{D}}=\gamma\left(\operatorname{cor}_{H}^{G}([D])\right)=\operatorname{cor}_{H}^{G}(\gamma([D]))=\operatorname{cor}_{H}^{G}\left(h_{D}\right)$. Now, by (2.3), $\Gamma_{D} / \Gamma_{F}=\operatorname{im}\left(h_{D}\right)$ and $\Gamma_{{ } D} / \Gamma_{F}=\operatorname{im}\left(h_{c_{D}}\right)$. But $\operatorname{im}\left(h_{c_{D}}\right)=$ $\operatorname{im}\left(\operatorname{cor}_{H}^{G}\left(h_{D}\right)\right) \subseteq \operatorname{im}\left(h_{D}\right)$ by $(1.3)$, as $G$ acts trivially on $\Delta / \Gamma_{F}$. Hence $\Gamma_{c_{D}} \subseteq \Gamma_{D}$.

(c) As $[D] \in \operatorname{Br}\left(L_{n r} / L\right)=\operatorname{Br}\left(F_{n r} / L\right)=H_{c}^{2}\left(H, F_{n r}^{*}\right)$, and [ $\left.{ }^{c} D\right] \epsilon$ $\operatorname{Br}\left(F_{n r} / F\right)=H_{c}^{2}\left(G, F_{n r}^{*}\right)$, by $(2.3), \mathcal{F}\left(\operatorname{ker}\left(h_{D}\right)\right)$ and $\mathcal{F}\left(\operatorname{ker}\left(h_{c_{D}}\right)\right)$ are the inertial lift of $Z(\bar{D})$ and $Z\left(\overline{{ }^{c} D}\right)$ respectively. Let $Z=\mathcal{F}\left(\operatorname{ker}\left(h_{D}\right)\right)$ and $M=\mathcal{N}(Z / F)$. Since $Z \subseteq F_{n r}$ and $F_{n r}$ is normal over $F, M \subseteq F_{n r}$. Let $I=\operatorname{Gal}\left(F_{n r} / M\right)$ and $K=\operatorname{Gal}\left(F_{n r} / Z\right)=\operatorname{ker}\left(h_{D}\right)$. Then $I$ is normal in $G=\operatorname{Gal}\left(F_{n r} / F\right)$, and $I \subseteq K \subseteq H=\operatorname{Gal}\left(F_{n r} / L\right) \subseteq G$. Note that $\operatorname{res}_{K}^{H}\left(h_{D}\right)=0$ as $K=\operatorname{ker}\left(h_{D}\right)$. Let $E$ be a set of representatives of the double cosets $I g H$. Then, by [B, p. 82, III, Prop. 3.5], $\operatorname{res}_{I}^{G}\left(\operatorname{cor}_{H}^{G}\left(h_{D}\right)\right)=$ $\sum_{g \in E} \operatorname{cor}_{I \cap g H^{-1}}^{I} \circ \operatorname{res}_{I \cap g H_{g^{-1}}}^{H}\left(h_{D}\right)$, where the "restriction map" on the right is with respect to the conjugation map $\left(i \mapsto g^{-1} i g, a \mapsto g^{-1} a\right)$, regarded as a map $\left(I \cap g H g^{-1}, F_{n r}^{*}\right) \stackrel{\cong}{\longrightarrow}\left(g^{-1} I g \cap H, F_{n r}^{*}\right) \rightarrow\left(H, F_{n r}^{*}\right)$. But as $I$ is normal in $G, g^{-1} I z g \cap H=I \cap H \subseteq K$, so $\operatorname{res}_{I \cap g H^{-1}}^{H}\left(h_{D}\right)=0$ for each $g \in E$. Hence, $\operatorname{res}_{I}^{G}\left(\operatorname{cor}_{H}^{G}\left(h_{D}\right)\right)=0$, so $\operatorname{ker}\left(h_{c}\right) \supseteq I$. Therefore $Z\left(\overline{{ }^{c} D}\right)=\overline{\mathcal{F}\left(\operatorname{ker}\left(h_{\left.\mathrm{c}_{D}\right)}\right)\right.} \subseteq \overline{\mathcal{F}(I)}=\bar{M}=\mathcal{N}(Z(\bar{D}) / \bar{F})$. 
(d) Since $D \in \mathcal{D}_{i}(L),[D] \in I \operatorname{Br}(L)=H_{c}^{2}(H, U)=\operatorname{ker}(\gamma)$, where $\gamma$ is the map of (2.1). Since the diagram above in the proof of (b) is commutative, $\left[{ }^{c} D\right]=\operatorname{cor}_{H}^{G}([D]) \in \operatorname{ker}(\gamma)=H_{c}^{2}(G, U)=I \mathrm{Br}(F)$. Hence ${ }^{c} D \in$ $\mathcal{D}_{i}(F)$. As the corestriction map is functorial, the map $\alpha$ in (2.2) is compatible with corestriction. So, since the algebraic corestriction corresponds to the homological corestriction, and by $(2.2)$, we have ${ }^{\bar{c} D} \sim \operatorname{cor}_{\bar{L} / \bar{F}}(\bar{D})$ in $\operatorname{Br}(\bar{F})$.

\section{Tame and totally ramified division algebras.}

We begin this section by giving a proposition we will use a number of times.

Proposition 3.1. Let $(F, v)$ be a Henselian valued field. For $1 \leq i \leq k$, let $n_{i} \geq 1$ be integers with $\left(\operatorname{char} \bar{F}, n_{i}\right)=1$, and $a_{i}, b_{i} \in F^{*}$. Assume $F$ has primitive $n_{i}$-th roots of unity $\omega_{i}$. Let $n=n_{1} \cdots n_{k}$ and $\ell=\ell \mathrm{cm}\left(n_{1}, \ldots, n_{k}\right)$. Then

(1) The tensor product of symbol algebras, $T=\bigotimes_{i=1}^{k}\left(a_{i}, b_{i} ; F\right)_{n_{i}}$ is a tame and totally ramified division algebra over $F$ if and only if

$$
\left\{\left(\ell / n_{i}\right) v\left(a_{i}\right),\left(\ell / n_{i}\right) v\left(b_{i}\right) \mid 1 \leq i \leq k\right\}
$$

generates a subgroup of order $n^{2}$ in $\Gamma_{F} / \ell \Gamma_{F}$; when this occurs,

$$
\Gamma_{T}=\left\langle\left(1 / n_{i}\right) v\left(a_{i}\right),\left(1 / n_{i}\right) v\left(b_{i}\right)\right\rangle_{i=1}^{k}+\Gamma_{F} .
$$

(2) If $D \in \mathcal{D}_{t t r}(F)$ and $D^{m}$ is the underlying division algebra of $D^{\otimes m}$, the $m$-fold tensor product of $D$ with itself over $F$, then $D^{m} \in \mathcal{D}_{t t r}(F)$, and

$$
\Gamma_{D^{m}} / \Gamma_{F}=m\left(\Gamma_{D} / \Gamma_{F}\right), \quad \text { so } \quad \Gamma_{D^{m}}=m \Gamma_{D}+\Gamma_{F} \text {. }
$$

Proof. (1) Suppose $\left\{\left(\ell / n_{i}\right) v\left(a_{i}\right)\left(\ell / n_{i}\right) v\left(b_{i}\right)\right\}_{i=1}^{k}$ generates a subgroup of or$\operatorname{der} n^{2}$ in $\Gamma_{F} / \ell \Gamma_{F}$. Then by [JW1, Cor. 2.6], $T \in \mathcal{D}_{t t r}(F)$ and $\Gamma_{T}=$ $\left\langle\frac{1}{n_{i}} v\left(a_{i}\right), \frac{1}{n_{i}} v\left(b_{i}\right)\right\rangle_{i=1}^{k}+\Gamma_{F}$. Conversely, suppose $T=\stackrel{\otimes}{i=1}_{i}^{k}\left(a_{i}, b_{i} ; F\right)_{n_{i}} \in \mathcal{D}_{t t r}(F)$. Let $\alpha_{i}, \beta_{i}$ be the generators of $T$ over $F$ with relations: $\alpha_{i}^{n_{i}}=a_{i}, \beta_{i}^{n_{i}}=b_{i}$, and $\alpha_{i} \beta_{i}=\omega_{i} \beta_{i} \alpha_{i}$. Then $\mathcal{A}=\left\langle\alpha_{i} F^{*}, \beta_{i} F^{*}\right\rangle_{i=1}^{k}$ is an armature of $T$, that is, an abelian subgroup of $T^{*} / F^{*}$ of order $\operatorname{dim}_{F} T$ such that $\left\{\alpha_{i}, \beta_{i}\right\}_{i=1}^{k}$ generates $T$ as an $F$-algebra. Since $T \in \mathcal{D}_{t t r}(F)$, by [TW, Prop. 3.3], the map $\bar{v}: \mathcal{A} \rightarrow \Gamma_{T} / \Gamma_{F}$ induced by $v$ (i.e., the map $x F^{*} \mapsto v(x)+$ $\left.\Gamma_{F}\right)$ is an isomorphism. So, $\Gamma_{T} / \Gamma_{F}=\left\langle\frac{1}{n_{i}} v\left(a_{i}\right)+\Gamma_{F}, \frac{1}{n_{i}} v\left(b_{i}\right)+\Gamma_{F}\right\rangle_{i=1}^{k}$ and 
$\left\{\left(\ell / n_{i}\right) v\left(a_{i}\right),\left(\ell / n_{i}\right) v\left(b_{i}\right)\right\}_{i=1}^{k}$ generates a subgroup of order $n^{2}$ in $\Gamma_{F} / \ell \Gamma_{F}$ (namely $\ell \Gamma_{T} / \ell \Gamma_{F}$ ), as $\left|\Gamma_{T} / \Gamma_{F}\right|=[T: F]=n^{2}$. Also,

$$
\Gamma_{T}=\left\langle\frac{1}{n_{i}} v\left(a_{i}\right), \frac{1}{n_{i}} v\left(b_{i}\right)\right\rangle_{i=1}^{k}+\Gamma_{F}
$$

as $\Gamma_{T} / \Gamma_{F}=\left\langle\frac{1}{n_{i}} v\left(a_{i}\right)+\Gamma_{F}, \frac{1}{n_{i}} v\left(b_{i}\right)+\Gamma_{F}\right\rangle_{i=1}^{k}$.

(2) As $D \in \mathcal{D}_{t t r}(F)$ and $F$ is Henselian, by Prop. 1.6 $D=\bigotimes_{i=1}^{k}\left(a_{i}, b_{i} ; F\right)_{n_{i}}$ for some $a_{i}, b_{i} \in F^{*}$.

Let $d_{i}=\operatorname{gcd}\left(n_{i}, m\right)$ and $n_{i}=d_{i} n_{i}^{\prime}, m=d_{i} m_{i}^{\prime}$ so that $\operatorname{gcd}\left(n_{i}^{\prime}, m_{i}^{\prime}\right)=1$. Since $D=\stackrel{\bigotimes}{i=1}_{\otimes}^{k}\left(a_{i}, b_{i} ; F\right)_{n_{i}} \in \mathcal{D}_{t t r}(F)$, by (1),

$$
\left\{\left(\ell / n_{i}\right) v\left(a_{i}\right),\left(\ell / n_{i}\right) v\left(b_{i}\right) \mid 1 \leq i \leq k\right\}
$$

generates a subgroup of order $n^{2}$ in $\Gamma_{F} / \ell \Gamma_{F}$. Also, since the map

$$
\Gamma_{F} / \ell \Gamma_{F} \rightarrow \frac{1}{\ell} \Gamma_{F} / \Gamma_{F},
$$

induced by multiplication of $\frac{1}{\ell}$, is an isomorphism,

$$
\left\{\frac{1}{n_{i}} v\left(a_{i}\right), \frac{1}{n_{i}} v\left(b_{i}\right) \mid 1 \leq i \leq k\right\}
$$

generates a subgroup $\left\langle\frac{1}{n_{i}} v\left(a_{i}\right)+\Gamma_{F}, \frac{1}{n_{i}} v\left(b_{i}\right)+\Gamma_{F} \mid 1 \leq i \leq k\right\rangle$ of order $n^{2}$ in $\frac{1}{\ell} \Gamma_{F} / \Gamma_{F}$. We want to show $\underset{i=1}{\otimes}\left(a_{i}, b_{i}^{m_{i}^{\prime}} ; F\right)_{n_{i}^{\prime}} \in \mathcal{D}_{t t r}(F)$. Since $n_{i}^{\prime} \mid n_{i}, 1 \leq i \leq$ $k,\left\langle\frac{1}{n_{i}^{\prime}} v\left(a_{i}\right)+\Gamma_{F}, \frac{1}{n_{i}^{\prime}} v\left(b_{i}\right)+\Gamma_{F}\right\rangle_{i=1}^{k}$ is a subgroup of

$$
\left\langle\frac{1}{n_{i}} v\left(a_{i}\right)+\Gamma_{F}, \frac{1}{n_{i}} v\left(b_{i}\right)+\Gamma_{F}\right\rangle_{i=1}^{k} .
$$

Since $\left|\left\langle\frac{1}{n_{i}} v\left(a_{i}\right)+\Gamma_{F}, \frac{1}{n_{i}} v\left(b_{i}\right)+\Gamma_{F} \mid 1 \leq i \leq k\right\rangle\right|=n^{2}$,

$$
\left|\left\langle\frac{1}{n_{i}^{\prime}} v\left(a_{i}\right)+\Gamma_{F}, \frac{1}{n_{i}^{\prime}} v\left(b_{i}\right)+\Gamma\right\rangle_{i=1}^{k}\right|=n^{\prime 2},
$$

where $n^{\prime}=n_{1}^{\prime} \cdots n_{k}^{\prime}$. Let $\ell^{\prime}=\ell \mathrm{cm}\left(n_{1}^{\prime}, \ldots, n_{k}^{\prime}\right)$. Then

$$
\left\langle\frac{1}{n_{i}^{\prime}} v\left(a_{i}\right)+\Gamma_{F}, \frac{1}{n_{i}^{\prime}} v\left(b_{i}\right)+\Gamma_{F}\right\rangle_{i=1}^{k} \subseteq \frac{1}{\ell^{\prime}} \Gamma_{F} / \Gamma_{F} .
$$


Since the map $\frac{1}{\ell^{\prime}} \Gamma_{F} / \Gamma_{F} \rightarrow \Gamma_{F} / \ell^{\prime} \Gamma_{F}$ induced by multiplication of $\ell^{\prime}$, is an isomorphism, $\left|\left\langle\frac{\ell^{\prime}}{n_{i}^{\prime}} v\left(a_{i}\right)+\ell^{\prime} \Gamma_{F}, \frac{\ell^{\prime}}{n_{i}^{\prime}} v\left(b_{i}\right)+\ell^{\prime} \Gamma_{F}\right\rangle_{i=1}^{k}\right|=n^{\prime 2}$. Also, since $\operatorname{gcd}\left(n_{i}^{\prime}, m_{i}^{\prime}\right)=1$,

$$
\left\{\left(\ell^{\prime} / n_{i}^{\prime}\right) v\left(a_{i}\right),\left(\ell^{\prime} / n_{i}^{\prime}\right) v\left(b_{i}^{m_{i}^{\prime}}\right) \mid 1 \leq i \leq k\right\}
$$

and

$$
\left\{\left(\ell^{\prime} / n_{i}^{\prime}\right) v\left(a_{i}\right),\left(\ell^{\prime} / n_{i}^{\prime}\right) v\left(b_{i}\right) \mid 1 \leq i \leq k\right\}
$$

generate the same subgroup of $\Gamma_{F} / \ell^{\prime} \Gamma_{F}$. So,

$$
\left\{\left(\ell^{\prime} / n_{i}^{\prime}\right) v\left(a_{i}\right),\left(\ell^{\prime} / n_{i}^{\prime}\right) v\left(b_{i}^{m_{i}^{\prime}}\right) \mid 1 \leq i \leq k\right\}
$$

generates a subgroup of order $n^{\prime 2}$ in $\Gamma_{F} / \ell^{\prime} \Gamma_{F}$. By applying (1) we have $\bigotimes_{i=1}^{k}\left(a_{i}, b_{i}^{m_{i}^{\prime}} ; F\right)_{n_{i}^{\prime}} \in \mathcal{D}_{t t r}(F)$ with value group

$$
\left\langle\left(1 / n_{i}^{\prime}\right) v\left(a_{i}\right),\left(1 / n_{i}^{\prime}\right) v\left(b_{i}^{m_{i}^{\prime}}\right)\right\rangle_{i=1}^{k}+\Gamma_{F}
$$

Since $D^{m} \sim \bigotimes_{i=1}^{k}\left(a_{i}, b_{i}^{m} ; F\right)_{n_{i}} \sim \bigotimes_{i=1}^{k}\left(a_{i}, b_{i}^{m^{\prime}} ; F\right)_{n_{i}^{\prime}}$, we have

$$
D^{m} \cong \bigotimes_{i=1}^{k}\left(a_{i}, b_{i}^{m_{i}^{\prime}} ; F\right)_{n_{i}^{\prime}} \in \mathcal{D}_{t t r}(F) .
$$

Because $\operatorname{gcd}\left(n_{i}^{\prime}, m_{i}^{\prime}\right)=1$, we have

$$
\begin{gathered}
\left\langle\left(1 / n_{i}^{\prime}\right) v\left(b_{i}\right)+\Gamma_{F}\right\rangle=\left\langle\left(m_{i}^{\prime} / n_{i}^{\prime}\right) v\left(b_{i}\right)+\Gamma_{F}\right\rangle= \\
=\left\langle\left(m / n_{i}\right) v\left(b_{i}\right)+\Gamma_{F}\right\rangle, \text { so } \Gamma_{D^{m}} / \Gamma_{F}= \\
=\left\langle\left(1 / n_{i}^{\prime}\right) v\left(a_{i}\right)+\Gamma_{F},\left(1 / n_{i}^{\prime}\right) v\left(b_{i}^{m_{i}^{\prime}}\right)+\Gamma_{F}\right\rangle_{i=1}^{k}= \\
=\left\langle\left(1 / n_{i}^{\prime}\right) v\left(a_{i}\right)+\Gamma_{F},\left(1 / n_{i}^{\prime}\right) v\left(b_{i}\right)+\Gamma_{F}\right)_{i=1}^{k}=m\left(\Gamma_{D} / \Gamma_{F}\right) .
\end{gathered}
$$

(In fact, the equality $\Gamma_{D^{m}}=m \Gamma_{D}+\Gamma_{F}$ is a special case of [JW2, Prop. 6.9].)

Recall that we assume that $(L, v) \supseteq(F, v)$ is a finite (separable) inertial extension of Henselian fields. Given any positive integer $n$ and a field $F$, we write $\mu_{n} \subseteq F$ to say that $F$ contains $n$ distinct $n$-th roots of unity.

Proposition 3.2. Let $T \in \mathcal{D}_{t t r}(L)$ and $n=\exp \left(\Gamma_{T} / \Gamma_{L}\right)$. If $\mu_{n} \subseteq F$, then $\Gamma_{\mathrm{c}_{T}} \subseteq \Gamma_{T}$.

Remark. Without the assumption $\mu_{n} \subseteq F$, it is still true $\Gamma_{c_{T}} \subseteq \Gamma_{T}$. We will prove this in Theorem 4.6 below. 
Proof. First we will show that if $T=(\alpha, \beta ; L)_{n}$ is a symbol algebra in $\mathcal{D}_{t t r}(L)$, then $[L: F] \Gamma_{T}+\Gamma_{F} \subseteq \Gamma_{c T} \subseteq \Gamma_{T}$ : Since $\Gamma_{L}=\Gamma_{F}, \alpha=a u$ and $\beta=b w$ for some $a, b \in F^{*}$ and $v$-units $u, w \in L^{*}$. Let $T_{0}=(a, b ; L)_{n}, A=(a, b ; F)_{n}$, and let $S$ be the underlying division algebra of $(a, w ; L)_{n} \otimes_{L}(u, \beta ; L)_{n}$. Since $T=(\alpha, \beta ; L)_{n} \in \mathcal{D}_{t t r}(L)$, by Prop. $3.1\{v(\alpha), v(\beta)\}$ generates a subgroup of order $n^{2}$ in $\Gamma_{L} / n \Gamma_{L}$, and $\Gamma_{T}=\langle(1 / n) v(\alpha),(1 / n) v(\beta)\rangle+\Gamma_{L}$. As $v(a)=v(\alpha)$, $v(b)=v(\beta)$ and $\Gamma_{F}=\Gamma_{L}$, by Prop. 3.1, again, $T_{0} \in \mathcal{D}_{t t r}(L)$ and $A \in \mathcal{D}_{t t r}(F)$ with

$$
\Gamma_{A}=\langle(1 / n) v(a),(1 / n) v(b)\rangle+\Gamma_{F}=\Gamma_{T_{0}}=\Gamma_{T} .
$$

Clearly $S \in \mathcal{D}_{i s}(L)$. So $T \sim T_{0} \otimes_{L} S$ in $\operatorname{Br}(L)$ with $T_{0} \in \mathcal{D}_{t t r}(L), S \in$ $\mathcal{D}_{i s}(L)$. Then by Prop. 1.7, $\Gamma_{T}=\Gamma_{T_{0}}+\Gamma_{S}$. Note that as $T_{0}=A \otimes_{F}$ $L,{ }^{c} T_{0} \sim A^{\otimes[L: F]}$ in $\mathrm{Br}(F)$, so ${ }^{c} T_{0}$ is the underlying division algebra of $A^{\otimes[L: F]}$, where $A \in \mathcal{D}_{t t r}(F)$. Then by Prop. $3.1(2),{ }^{c} T_{0} \in \mathcal{D}_{t t r}(F)$, and $\Gamma_{{ }^{c} T_{0}}=[L: F] \Gamma_{A}+\Gamma_{F}=[L: F] \Gamma_{T}+\Gamma_{F}$ as $\Gamma_{T}=\Gamma_{A}$. Also, by Theorem 2.4, ${ }^{c} S \in \mathcal{D}_{i s}(F)$ and $\Gamma_{{ }^{c} S} \subseteq \Gamma_{S}$. Since ${ }^{c} T \sim{ }^{c} T_{0} \otimes_{F}{ }^{c} S$ in $\operatorname{Br}(F)$ where ${ }^{c} T_{0} \in \mathcal{D}_{t t r}(F)$ and ${ }^{c} S \in \mathcal{D}_{i s}(F)$, by Prop. 1.7, $\Gamma_{c_{T}}=\Gamma_{c T_{0}}+\Gamma_{c_{S}}$. Hence $[L: F] \Gamma_{T}+\Gamma_{F} \subseteq \Gamma_{c T_{0}}+\Gamma_{c_{S}}=\Gamma_{c T} \subseteq \Gamma_{T_{0}}+\Gamma_{S}=\Gamma_{T}$, as desired.

Now, if $T \in \mathcal{D}_{t t r}(L)$, then by Prop. 1.6, $T \cong \stackrel{\otimes}{i=1}_{i}^{k} T_{i}$ where each $T_{i}$ is a symbol algebra in $\mathcal{D}_{t t r}(L)$ of index $n_{i}$ with $n_{i} \mid n$. Also, $\Gamma_{T}=\sum_{i=1}^{k} \Gamma_{T_{i}}$. Then ${ }^{c} T \sim \stackrel{\otimes}{\otimes}^{k} T_{i}$ in $\operatorname{Br}(F)$. Since each ${ }^{c} T_{i} \in \mathcal{D}_{t}(F)$ by Theorem 2.4, $\Gamma_{c_{T}} \subseteq \sum_{i=1}^{k} \Gamma_{{ }^{\prime} T_{i}}$ by [JW2, Cor. 6.7]. Since $\Gamma_{{ }^{\prime} T_{i}} \subseteq \Gamma_{T_{i}}$ by what we have shown above, $\Gamma_{c T} \subseteq \sum_{i=1}^{k} \Gamma_{c T_{\imath}} \subseteq \sum_{i=1}^{k} \Gamma_{T_{i}}=\Gamma_{T}$.

We will shortly give Proposition 3.4, which will be very useful for this paper. The following purely group-theoretic lemma will be used in the proof of that proposition.

Lemma 3.3. Let $A_{1} \times A_{2}$ be the direct product of cyclic groups $A_{i}$ of order $r_{i}, i=1,2$. Let $\pi_{i}$ be the projection map of $A_{1} \times A_{2}$ onto $A_{i}$ for $i=1,2$. Let $H$ be a subgroup of $A_{1} \times A_{2}$ such that $\pi_{i}(H)=A_{i}, i=1,2$. Let $B_{1}=H \cap A_{2}$ and $B_{2}=H \cap A_{1}$. If $t$ is any positive integer and $T$ is the $t$-torsion subgroup of $H$, then $T=C_{1} \cap C_{2}$, where $C_{i}, i=1,2$, are the unique subgroups of $H$ such that $C_{i} \supseteq B_{i}$ and $\left|C_{i}: B_{i}\right|=\operatorname{gcd}\left(r_{i}, t\right)$.

Proof. Since $\left|C_{i}: B_{i}\right|$ divides $t, t\left(C_{i} / B_{i}\right)=(0)$, so $t C_{i} \subseteq B_{i}$. Hence $t\left(C_{1} \cap\right.$ $\left.C_{2}\right) \subseteq B_{1} \cap B_{2}=(0)$, and $C_{1} \cap C_{2} \subseteq T$. Take $h \in H$ with $t h=0$ (i.e., $h \in T)$. Then $0=\pi_{i}(t h)=t \pi_{i}(h)$. But $r_{i} \pi_{i}(h)=0$ as $\left|A_{i}\right|=r_{i}$. So $\operatorname{gcd}\left(r_{i}, t\right) \cdot \pi_{i}(h)=0$. Let $D_{i}$ be the subgroup of $A_{i}$ of order $\operatorname{gcd}\left(r_{i}, t\right)$. Then 
$\pi_{i}(h) \in D_{i}$ as $A_{i}$ is cyclic. Since $B_{i}=\operatorname{ker}\left(\left.\pi_{i}\right|_{H}\right)$ and $\left.\pi_{i}\right|_{H}$ is onto, the map $\tilde{\pi}_{i}: H / B_{i} \rightarrow A_{i}$ induced by $\left.\pi_{i}\right|_{H}$ is an isomorphism. Since $\tilde{\pi}_{i}\left(h+B_{i}\right)=$ $\pi_{i}(h) \in D_{i}, h+B_{i} \in \tilde{\pi}_{i}^{-1}\left(D_{i}\right)$. But $\tilde{\pi}_{i}^{-1}\left(D_{i}\right)=C_{i} / B_{i}$, for $i=1,2$. Hence $h \in C_{1} \cap C_{2}$. Therefore, $T \subseteq C_{1} \cap C_{2}$.

Proposition 3.4. Let $(F, v)$ be a Henselian field. Let $r_{1}, r_{2}$ and $t$ be positive integers, and let $M_{i} / F, i=1,2$, be cyclic inertial extensions of degree $r_{i}$ with $\operatorname{Gal}\left(M_{i} / F\right)=\left\langle\tau_{i}\right\rangle$. Let $x_{1}, x_{2} \in F^{*}$ such that, for any prime $p$ dividing $r_{1}, r_{2}$ or $t, v\left(x_{1}\right)+p \Gamma_{F}, v\left(x_{2}\right)+p \Gamma_{F}$ are $\mathbb{Z}_{p}$-linearly independent in $\Gamma_{F} / p \Gamma_{F}$. Suppose $D$ is the underlying division algebra of $\left(M_{1} / F, \tau_{1}, x_{1}\right)_{r_{1}} \otimes_{F}\left(M_{2} / F, \tau_{2}, x_{2}\right)_{r_{2}} \otimes_{F}\left(x_{1}, x_{2} ; F\right)_{t}$. Then $Z(\bar{D})=\widetilde{E}_{1} \widetilde{E}_{2}$, where $\overline{M_{i}} \supseteq \widetilde{E}_{i} \supseteq \bar{F}$, and $\left[\overline{M_{i}}: \widetilde{E}_{i}\right]=\operatorname{gcd}\left(r_{i}, t\right)$ for $i=1,2$.

Proof. Let $\Delta$ be the divisible hull of $\Gamma_{F}$ and let $r_{1}=p_{1}^{e_{1}} p_{2}^{e_{2}} \cdots p_{k}^{e_{k}}, r_{2}=$ $p_{1}^{f_{1}} p_{2}^{f_{2}} \cdots p_{k}^{f_{k}}$ and $t=p_{1}^{t_{1}} p_{2}^{t_{2}} \cdots p_{k}^{t_{k}}$, where the $p_{i}$ are distinct primes and $e_{i}$, $f_{i}, t_{i} \geq 0$ such that at least one of $e_{i}, f_{i}$ and $t_{i}$ is larger than 0 for all $i$, $1 \leq i \leq k$. Since $v\left(x_{1}\right)+p_{i} \Gamma_{F}, v\left(x_{2}\right)+p_{i} \Gamma_{F}$ are $\mathbb{Z}_{p_{i}}$-linearly independent in $\Gamma_{F} / p_{i} \Gamma_{F}$ by assumption, in $\Delta / \Gamma_{F}$,

$$
\left|\left\langle\frac{1}{p_{i}^{e_{i}}} v\left(x_{1}\right)+\Gamma_{F}, \frac{1}{p_{i}^{f_{i}}} v\left(x_{2}\right)+\Gamma_{F}\right\rangle\right|=p_{i}^{e_{i}+f_{i}}
$$

and

$$
\left|\left\langle\frac{1}{p_{i}^{t_{i}}} v\left(x_{1}\right)+\Gamma_{F}, \frac{1}{p_{i}^{t_{i}}} v\left(x_{2}\right)+\Gamma_{F}\right\rangle\right|=p_{i}^{2 t_{i}} .
$$

Then in $\Delta / \Gamma_{F}$,

$$
\left|\left\langle\frac{1}{r_{1}} v\left(x_{1}\right)+\Gamma_{F}, \frac{1}{r_{2}} v\left(x_{2}\right)+\Gamma_{F}\right\rangle\right|=r_{1} r_{2}
$$

and

$$
\left|\left\langle\frac{1}{t} v\left(x_{1}\right)+\Gamma_{F}, \frac{1}{t} v\left(x_{2}\right)+\Gamma_{F}\right\rangle\right|=t^{2} .
$$

Let $T=\left(x_{1}, x_{2} ; F\right)_{t}$ and $S_{i}=\left(M_{i} / F, \tau_{i}, x_{i}\right)_{r_{i}}$ for $i=1,2$. Then by Prop. 3.1, $T \in \mathcal{D}_{t t r}(F)$. Also, by [JW1, Cor.2.9], $S_{i} \in \mathcal{D}(F)$ with $\overline{S_{i}}=\overline{M_{i}}$ and $\Gamma_{S_{i}}=\left\langle\frac{1}{r_{i}} v\left(x_{i}\right)\right\rangle+\Gamma_{F}$ for $i=1,2$. Also, $S_{i}$ is inertially split over $F$ as $M_{i}$ is a splitting field of $S_{i}$. Let $S$ be the underlying division algebra of $S_{1} \otimes_{F} S_{2}$. Then $D \sim S \otimes_{F} T$ in $\operatorname{Br}(F)$ where $S \in \mathcal{D}_{i s}(F)$ and $T \in \mathcal{D}_{t t r}(F)$. Let $h_{S}$, $h_{S_{1}}, h_{S_{2}} \in \operatorname{Hom}_{c}\left(G_{F}, \Delta / \Gamma_{F}\right)$ as in (2.3). Since $h_{S}=h_{S_{1}}+h_{S_{2}}, \operatorname{im}\left(h_{S_{i}}\right)=$ $\Gamma_{S_{i}} / \Gamma_{F}=\left\langle\frac{1}{r_{i}} v\left(x_{i}\right)+\Gamma_{F}\right\rangle, i=1,2$, and $\left\langle\frac{1}{r_{1}} v\left(x_{1}\right)+\Gamma_{F}\right\rangle \cap\left\langle\frac{1}{r_{2}} v\left(x_{2}\right)+\Gamma_{F}\right\rangle=$ (0) as $\left|\left\langle\frac{1}{r_{1}} v\left(x_{1}\right)+\Gamma_{F}, \frac{1}{r_{2}} v\left(x_{2}\right)+\Gamma_{F}\right\rangle\right|=r_{1} r_{2}$, we have $\operatorname{ker}\left(h_{S}\right)=\operatorname{ker}\left(h_{S_{1}}\right) \cap$ 
$\operatorname{ker}\left(h_{S_{2}}\right)$. So by $(2.3)$,

$$
\begin{aligned}
Z(\bar{S}) & \left.\left.\left.=\overline{\mathcal{F}\left(\operatorname{ker} h_{S}\right.}\right)=\overline{\mathcal{F}\left(\operatorname{ker} h_{S_{1}}\right.}\right) \cdot \overline{\mathcal{F}\left(\operatorname{ker} h_{S_{2}}\right.}\right) \\
& =Z\left(\overline{S_{1}}\right) Z\left(\overline{S_{2}}\right)=\overline{M_{1}} \cdot \overline{M_{2}} .
\end{aligned}
$$

Also,

$$
\begin{aligned}
\Gamma_{S} / \Gamma_{F}=\operatorname{im}\left(h_{S}\right) \subseteq & \operatorname{im}\left(h_{S_{1}}\right)+\operatorname{im}\left(h_{S_{2}}\right)= \\
& =\left\langle\frac{1}{r_{1}} v\left(x_{1}\right)+\Gamma_{F}\right\rangle \times\left\langle\frac{1}{r_{2}} v\left(x_{2}\right)+\Gamma_{F}\right\rangle \cong \mathbb{Z}_{r_{1}} \times \mathbb{Z}_{r_{2}} .
\end{aligned}
$$

Since $S \in \mathcal{D}_{i s}(F)$, by [JW2, Lemma 5.1], $\theta_{S}: \Gamma_{S} / \Gamma_{F} \rightarrow \operatorname{Gal}(Z(\bar{S}) / \bar{F})$ is an isomorphism. Let $H=\Gamma_{S} / \Gamma_{F}$ and $A_{i}=\Gamma_{S_{i}} / \Gamma_{F}$ for $i=1,2$. Then $H \subseteq A_{1} \times A_{2}$. Let $B_{1}=H \cap A_{2}$ and $B_{2}=H \cap A_{1}$. Since $H=\operatorname{im}\left(h_{S}\right)=$ $\operatorname{im}\left(h_{S_{1}}+h_{S_{2}}\right)$, and $\operatorname{im}\left(h_{S_{1}}\right) \cap \operatorname{im}\left(h_{S_{2}}\right)=(0), \pi_{i}(H)=A_{i}$ where $\pi_{i}: A_{1} \times A_{2} \rightarrow$ $A_{i}$ is the $i$-th projection map. Note that

$$
\begin{aligned}
& \left(\Gamma_{S} \cap \Gamma_{T}\right) / \Gamma_{F}=\left(\Gamma_{S} / \Gamma_{F}\right) \cap\left\langle\frac{1}{t} v\left(x_{1}\right)+\Gamma_{F}, \frac{1}{t} v\left(x_{2}\right)+\Gamma_{F}\right\rangle= \\
= & \left\{\alpha \in \Gamma_{S} / \Gamma_{F} \mid t \alpha=0\right\} \text { as } \Gamma_{S} / \Gamma_{F} \subseteq\left\langle\frac{1}{r_{1}} v\left(x_{1}\right)+\Gamma_{F}\right\rangle \times\left\langle\frac{1}{r_{2}} v\left(x_{2}\right)+\Gamma_{F}\right\rangle .
\end{aligned}
$$

So $\left(\Gamma_{S} \cap \Gamma_{T}\right) / \Gamma_{F}$ is the $t$-torsion subgroup of $H$. Then by Lemma 3.3, $\left(\Gamma_{S} \cap \Gamma_{T}\right) / \Gamma_{F}=C_{1} \cap C_{2}$, where $C_{i} \supseteq B_{i}$ and $\left|C_{i}: B_{i}\right|=\operatorname{gcd}\left(r_{i}, t\right)$ for $i=1,2$.

We have the map $h_{S}: \operatorname{Gal}\left(F_{n r} / F\right) \rightarrow \Gamma_{S} / \Gamma_{F}=H \subseteq A_{1} \times A_{2}$, where $A_{i}=\operatorname{im}\left(h_{S_{i}}\right)$ for $i=1,2$. Since $A_{1} \cap A_{2}=(0)$, for $g \in \operatorname{Gal}\left(F_{n r} / F\right)$, $A_{2}\left(=\operatorname{im}\left(h_{S_{2}}\right)\right)$ contains $h_{S}(g)=h_{S_{1}}(g)+h_{S_{2}}(g)$ if and only if $h_{S_{1}}(g)=$ 0 . So, $h_{S}^{-1}\left(B_{1}\right)=h_{S}^{-1}\left(G \cap A_{2}\right)=\operatorname{ker}\left(h_{S_{1}}\right)=\operatorname{Gal}\left(F_{n r} / M_{1}\right)$. Likewise, $h_{S}^{-1}\left(B_{2}\right)=\operatorname{ker}\left(h_{S_{2}}\right)=\operatorname{Gal}\left(F_{n r} / M_{2}\right)$. Hence, in the isomorphism $\theta_{S}=$ $\tilde{h}_{S}^{-1}: \Gamma_{S} / \Gamma_{F} \longrightarrow \operatorname{Gal}\left(\overline{M_{1} M_{2}} / \bar{F}\right), \theta_{S}\left(B_{i}\right)=\operatorname{Gal}\left(\overline{M_{1} M_{2}} / \overline{M_{i}}\right)$ for $i=1,2$. Therefore $\theta_{S}\left(C_{i}\right)=\operatorname{Gal}\left(\overline{M_{1} M_{2}} / \widetilde{E}_{i}\right)$ where $\overline{M_{i}} \supseteq \widetilde{E}_{i} \supseteq \bar{F}$ and $\left[\overline{M_{i}}: \widetilde{E}_{i}\right]=$ $\operatorname{gcd}\left(r_{i}, t\right)$ for $i=1,2$. Then by Prop. 1.7, $Z(\bar{D})=\mathcal{F}\left(\theta_{S}\left(\left(\Gamma_{S} \cap \Gamma_{T}\right) / \Gamma_{F}\right)\right)=$ $\mathcal{F}\left(C_{1} \cap C_{2}\right)=\widetilde{E}_{1} \widetilde{E}_{2}$.

Corollary 3.5. Let $(F, v)$ be a Henselian field. Let $r_{1}, r_{2}$, and $t$ be positive integers and assume $\mu_{t}, \mu_{r_{1}}, \mu_{r_{2}} \subseteq F$. Let $u_{1}, u_{2}$ be v-units in $F$, and let $x_{1}, x_{2} \in F^{*}$ such that for any prime $p$ dividing $r_{1}, r_{2}$ or $t, v\left(x_{1}\right)+p \Gamma_{F}$, $v\left(x_{2}\right)+p \Gamma_{F}$ are $\mathbb{Z}_{p}$-linearly independent in $\Gamma_{F} / p \Gamma_{F}$. Let $\omega_{t}\left(\right.$ resp. $\left.\omega_{r_{i}}\right)$ be an arbitrary primitive $t$-th (resp. $r_{i}$-th) root of unity in $F$. Suppose $D$ is the underlying division algebra of $\left(x_{1}, u_{1}, \omega_{r_{1}} ; F\right)_{r_{1}} \otimes_{F}\left(x_{2}, u_{2}, \omega_{r_{2}} ; F\right)_{r_{2}} \otimes_{F}$ $\left(x_{1}, x_{2}, \omega_{t} ; F\right)_{t}$. Then $Z(\bar{D})=\bar{F}\left(\bar{u}_{1}^{t / r_{1}}, \bar{u}_{2}^{t / r_{2}}\right)$.

Proof. Let $s_{i}=o\left(u_{i} F^{* r_{i}}\right)$ in $F^{*} / F^{* r_{i}}$ for $i=1,2$. Then $s_{i} \mid r_{i}, u_{i}^{s_{i}}=$ $w_{i}^{\prime r_{i}}$ for some $w_{i}^{\prime} \in F^{*}$, so $u_{i}=\omega_{s_{i}}^{k} w_{i}^{\prime r_{i} / s_{i}}=\left(\omega_{r_{i}}^{k} w_{i}^{\prime}\right)^{r_{i} / s_{i}}$ for some $k \in \mathbb{Z}$, 
where $\omega_{r_{i}}$ is the given primitive $r_{i}$-th root of unity, and $\omega_{s_{i}}=\omega_{r_{i}}^{r_{i} / s_{i}}$. Let $w_{i}=\omega_{r_{i}}^{k} w_{i}^{\prime}$. Then $u_{i}=w_{i}^{r_{i / s_{i}}}$ and $F\left(u_{i}^{1 / r_{i}}\right)=F\left(w_{i}^{1 / s_{i}}\right)$ is a cyclic inertial extension of $F$ of degree $s_{i}$ for each $i=1,2$. Also, $D \sim\left(x_{1}, w_{1}^{r_{1} / s_{1}} ; F\right)_{r_{1}} \otimes_{F}$ $\left(x_{2}, w_{2}^{r_{2} / s_{2}} ; F\right)_{r_{2}} \otimes_{F}\left(x_{1}, x_{2} ; F\right)_{t} \sim\left(x_{1}, w_{1} ; F\right)_{s_{1}} \otimes_{F}\left(x_{2}, w_{2} ; F\right)_{s_{2}} \otimes_{F}\left(x_{1}, x_{2} ; F\right)_{t}$. But $\left(x_{i}, w_{i} ; F\right)_{s_{i}}=\left(M_{i} / F, \tau_{i}, x_{i}\right)_{s_{i}}$ where $M_{i}=F\left(w_{i}^{1 / s_{i}}\right)$ are cyclic inertial over $F$ of degree $s_{i}$, and $\tau_{i}$ are some generators of Gal $\left(M_{i} / F\right)$. So, by Prop. $3.4, Z(\bar{D})=\widetilde{E}_{1} \widetilde{E}_{2}$ where $\overline{M_{i}} \supseteq \widetilde{E}_{i} \supseteq \bar{F}$, and $\left[\bar{M}_{i}: \widetilde{E}_{i}\right]=\operatorname{gcd}\left(s_{i}, t\right)$, so $\widetilde{E}_{i}=\bar{F}\left(\bar{w}_{i}^{t / s_{i}}\right)=\bar{F}\left(\bar{u}_{i}^{t / r_{i}}\right)$. Hence $Z(\bar{D})=\bar{F}\left(\bar{u}_{1}^{t / r_{1}}, \bar{u}_{2}^{t / r_{2}}\right)$.

We will consider $Z(\bar{c} T)$ for $T \in \mathcal{D}_{t t r}(L)$ in Theorem 3.9. We first introduce an equivalence relation in $\mathcal{D}_{t}(F)$ for a Henselian field $(F, v)$. For any $D_{1}, D_{2} \in \mathcal{D}_{t}(F)$, we write $D_{1} \approx D_{2}$ if $\left[D_{2}\right] \equiv\left[D_{1}\right] \bmod I \operatorname{Br}(F)$ in $\operatorname{Br}(F)$. This is an equivalence relation since $I \mathrm{Br}(F)$ is a subgroup of $\mathrm{Br}(F)$ (cf. [JW2, Prop. 2.5]).

Remark 3.6. Let $(F, v)$ be Henselian.

(i) If $D_{1}, D_{2} \in \mathcal{D}_{t}(F)$ and $D_{1} \approx D_{2}$, then $Z\left(\overline{D_{1}}\right)=Z\left(\overline{D_{2}}\right)$ by [JW2, Cor. 6.8].

(ii) If $L / F$ is inertial, $D_{1}, D_{2} \in \mathcal{D}_{t}(L)$ and $D_{1} \approx D_{2}$, then ${ }^{c} D_{1} \approx{ }^{c} D_{2}$ since ${ }^{c} I \in \mathcal{D}_{i}(F)$ for any $I \in \mathcal{D}_{i}(L)$ by Theorem $2.4(\mathrm{~d})$.

The following lemma will be used in proof of Prop. 3.8.

Lemma 3.7. Let $K / F$ be a finite extension of Henselian fields. If $\alpha$ is a $v$ unit in $K$, then $N_{K / F}(\alpha)$ is a v-unit in $F$, and $\overline{N_{K / F}(\alpha)}=N_{\bar{K} / \bar{F}}(\bar{\alpha})^{e}$ where $e=[K: F] /[\bar{K}: \bar{F}]$.

Proof. Recall that the integral closure of $V_{F}$ in $K$ is the intersection of all the valuation rings of $K$ extending $V_{F}$ (cf. [Bo, Ch. VI, Sec. 1, Th. 3, p. 378]). As $F$ is Henselian, $V_{K}$ is the unique valuation ring of $K$ extending $V_{F}$. So $V_{K}$ is the integral closure of $V_{F}$ in $K$. Hence $\alpha$ is integral over $V_{F}$. Let $f$ be the minimal polynomial of $\alpha$ over $F$. Then $f \in V_{F}[x]$ since $V_{F}$ is integrally closed.

Note that $\bar{f}=\varphi^{i}$ where $\varphi \in \bar{F}[X]$ is a monic irreducible polynomial, $i \geq 1$ and $i \operatorname{deg}(\varphi)=\operatorname{deg}(f)$ since $f \in V_{F}[X]$ is a monic irreducible polynomial and $F$ is Henselian (cf. [Rb, Th. 3]). Since $\varphi(\bar{\alpha})^{i}=\bar{f}(\bar{\alpha})=\overline{f(\alpha)}=0$, i.e., $\varphi(\bar{\alpha})=0, \varphi$ is the minimal polynomial of $\bar{\alpha}$ over $\bar{F}$. So $N_{\bar{K} / \bar{F}}(\bar{\alpha})=$ $(-1)^{[\bar{K}: \bar{F}]}(\varphi(0))^{[\bar{K}: \bar{F}] \operatorname{deg}}(\varphi)$ (cf. [L, Ch. VIII, Sec. 5, Th. 5.1]). On the other hand, $N_{K /}(\alpha)=(-1)^{[K: F]}(f(0))^{[K: F] \operatorname{deg}}(f)$. But since $\overline{f(0)}=\varphi(0)^{i}$ and $\operatorname{deg}(f)=i \operatorname{deg}(\varphi), \overline{N_{K / F}(\alpha)}=(-1)^{[K: F]}(\varphi(0))^{i[K: F] / i \operatorname{deg}}(\varphi)=N_{\bar{K} / \bar{F}}(\bar{\alpha})^{e}$ where $e=[K: F] /[\bar{K}: \bar{F}]$. 
Proposition 3.8. Let the symbol algebra $T=\left(\alpha_{1}, \alpha_{2} ; L\right)_{n} \in \mathcal{D}_{t t r}(L)$ where $n>1$ is any integer, (so $\mu_{n} \subseteq L$ ) and $\alpha_{1}, \alpha_{2} \in L^{*}$. If $\mu_{n} \subseteq F$, then $Z(\bar{c} \bar{T}) \subseteq \bar{F}\left(N_{\bar{L} / \bar{F}}(\bar{L})^{1 / d}\right)$, where $d=\operatorname{gcd}(n,[L: F])$.

Proof. Since $\Gamma_{L}=\Gamma_{F}, \alpha_{i}=a_{i} u_{i}$ for some $a_{i} \in F^{*}, u_{i} \in U_{L}$. Note that since $\left(\alpha_{1}, \alpha_{2} ; L\right)_{n} \in \mathcal{D}_{t t r}(L)$, by Prop. 3.1, in $\Gamma_{L} / n \Gamma_{L}$

$$
\left|\left\langle v\left(\alpha_{1}\right)+n \Gamma_{L}, v\left(\alpha_{2}\right)+n \Gamma_{L}\right\rangle\right|=n^{2} .
$$

But as $v\left(a_{i}\right)=v\left(\alpha_{i}\right), i=1,2$, and $\Gamma_{L} / n \Gamma_{L}=\Gamma_{F} / n \Gamma_{F} \cong \frac{1}{n} \Gamma_{F} / \Gamma_{F}$, we have in $\frac{1}{n} \Gamma_{F} / \Gamma_{F},\left|\left\langle\frac{1}{n} v\left(a_{1}\right)+\Gamma_{F}, \frac{1}{n} v\left(a_{2}\right)+\Gamma_{F}\right\rangle\right|=n^{2}$. So for any prime $p$ dividing $n$, in $\frac{1}{p} \Gamma_{F} / \Gamma_{F},\left|\left\langle\frac{1}{p} v\left(a_{1}\right)+\Gamma_{F} \frac{1}{p} v\left(a_{1}\right)+\Gamma_{F}\right\rangle\right|=p^{2}$, or equivalently in $\Gamma_{F} / p \Gamma_{F}, v\left(a_{1}\right)+p \Gamma_{F}, v\left(a_{2}\right)+p \Gamma_{F}$ are $\mathbb{Z}_{p}$-linearly independent.

Note that $\left[\left(u_{1}, u_{2} ; L\right)_{n}\right] \in I \operatorname{Br}(L)$ by [JW2, Prop. 2.5], since the "symbol algebra" $\left(u_{1}, u_{2} ; V_{L}\right)_{n}$ over the valuation ring $V_{L}$ is an Azumaya algebra over $V_{L}$ and an order in $\left(u_{1}, u_{2} ; L\right)_{n}$ (cf. [JW2, Ex. 2.4 (i)]). Then since $T \sim$ $\left(u_{1}, u_{2} ; L\right)_{n} \otimes\left(a_{1}, u_{2} ; L\right)_{n} \otimes\left(u_{1}, a_{2} ; L\right)_{n} \otimes\left(a_{1}, a_{2} ; L\right)_{n}$ in $\operatorname{Br}(L)$, we have $T \approx D$ where $D$ is the underlying division algebra of $\left(a_{1}, u_{2} ; L\right)_{n} \otimes_{L}\left(a_{2}, u_{1}^{-1} ; L\right)_{n} \otimes_{L}$ $\left(a_{1}, a_{2} ; L\right)_{n}$. By Remark 3.6 (ii), and Prop. 1.4, and as $N_{L / F}\left(a_{2}\right)=a_{2}^{[L: F]}$, ${ }^{c} T \approx{ }^{c} D \sim\left(a_{1}, N_{L / F}\left(u_{2}\right) ; F\right)_{n} \otimes_{F}\left(a_{2}, N_{L / F}\left(u_{1}^{-1}\right) ; F\right)_{n} \otimes_{F}\left(a_{1}, a_{2}^{[L: F]} ; F\right)_{n}$. But $\left(a_{1}, a_{2}^{[L: F]} ; F\right)_{n} \sim\left(a_{1}, a_{2}^{e} ; F\right)_{n^{\prime}}$, where $d=\operatorname{gcd}(n,[L: F]),[L: F]=d e$, and $n=d n^{\prime}$ so that $\operatorname{gcd}\left(e, n^{\prime}\right)=1$. Say, let $\left(a_{1}, a_{2}^{e} ; F\right)_{n^{\prime}}=\left(a_{1}, a_{2}^{e}, \omega ; F\right)_{n^{\prime}}$ for some primitive $n^{\prime}$-th root $\omega$ of unity. Then $\left(a_{1}, a_{2}^{e}, \omega ; F\right)_{n^{\prime}}=\left(a_{1}, a_{2}, \omega^{k} ; F\right)_{n^{\prime}}$ for some $k$ with $\operatorname{gcd}\left(k, n^{\prime}\right)=1$. So $\left(a_{1}, a_{2}^{[L: F]} ; F\right)_{n}$ is similar to a symbol algebra $\left(a_{1}, a_{2}, \omega^{k} ; F\right)_{n^{\prime}}$ where $n^{\prime}=n / \operatorname{gcd}(n,[L: F])$. Hence ${ }^{c} T \approx{ }^{c} D \sim$ $\left(a_{1}, N_{L / F}\left(u_{2}\right) ; F\right)_{n} \otimes_{F}\left(a_{2}, N_{L / F}\left(u_{1}^{-1}\right) ; F\right)_{n} \otimes\left(a_{1}, a_{2}, \omega^{k} ; F\right)_{n^{\prime}}$ in $\operatorname{Br}(F)$. Then by Remark 3.6, and Cor. 3.5,

$$
Z(\overline{c T})=Z(\overline{c D})=\bar{F}\left(\left(\overline{N_{L / F}\left(u_{2}\right)}\right)^{n^{\prime} / n},\left(\overline{N_{L / F}\left(u_{1}^{-1}\right)}\right)^{n^{\prime} / n}\right) .
$$

Since $[L: F]=[\bar{L}: \bar{F}], \overline{N_{L / F}\left(u_{1}^{-1}\right)}=N_{\bar{L} / \bar{F}}\left(\overline{\left.u_{1}^{-1}\right)}\right.$ and $\overline{N_{L / F}\left(u_{2}\right)}=N_{\bar{L} / \bar{F}}\left(\overline{u_{2}}\right)$ by Lemma 3.7 above. So

$$
Z(\overline{c T})=\bar{F}\left(N_{\bar{L} / \bar{F}}\left(\bar{u}_{2}\right)^{1 / d}, N_{\bar{L} / \bar{F}}\left(\overline{u_{1}^{-1}}\right)^{1 / d}\right) \subseteq \bar{F}\left(N_{\bar{L} / \bar{F}}(\bar{L})^{1 / d}\right)
$$

where $d=n / n^{\prime}=\operatorname{gcd}(n,[L: F])$.

Theorem 3.9. Suppose $T \in \mathcal{D}_{t t r}(L)$ and $n=\exp \left(\Gamma_{T} / \Gamma_{L}\right)$. (So $\mu_{n} \subseteq L$.) If $\mu_{n} \subseteq F$, then

$$
Z(\overline{c T}) \subseteq \bar{F}\left(N_{\bar{L} / \bar{F}}(\bar{L})^{1 / d}\right), d=\operatorname{gcd}(n,[L: F]),
$$


where $N_{\bar{L} / \bar{F}}$ is the norm map from $\bar{L}$ to $\bar{F}$.

Proof. By Prop. 1.6, $T=\stackrel{\bigotimes}{\gtrless}=1_{i} T_{i}$, where each $T_{i}$ is a symbol division algebra in $\mathcal{D}_{t t r}(L)$ of index $n_{i}$ with $n_{i} \mid n$. Then ${ }^{c} T \sim \otimes_{i=1}^{k}{ }^{c} T_{i} \sim{ }^{c} T_{0} \otimes_{F}{ }^{c} T_{k}$ where $T_{0}=\bigotimes_{i=1}^{k-1} T_{i}$. We prove that $Z(\overline{c T})=Z\left(\overline{{ }^{c} T_{1}}\right) \cdots Z\left(\overline{c T_{k}}\right)$. By induction on $k$, we need only to prove $Z\left(\overline{{ }^{c} T}\right)=Z\left(\overline{{ }^{c} T_{0}}\right) Z\left(\overline{c T_{k}}\right)$. Since ${ }^{c} T_{0} \in \mathcal{D}_{t}(F)$ by Theorem 2.4, ${ }^{c} T_{0} / F$ is defectless, and $Z\left(\overline{{ }^{c} T_{0}}\right) / \bar{F}$ is Galois by [JW2, Lemma 6.1]. So, $\mathcal{L}=Z\left(\overline{{ }^{c} T_{0}}\right) \cap Z\left(\overline{{ }^{c} T_{k}}\right)$ is separable over $\bar{F}$, and $Z\left(\overline{{ }^{c} T_{0}}\right)$ and $Z\left(\overline{{ }^{c} T_{k}}\right)$ are linearly disjoint over $\mathcal{L}$. Also, by Prop. 3.2, and as $\Gamma_{T} / \Gamma_{L}=\oplus_{i=1}^{k}\left(\Gamma_{T_{i}} / \Gamma_{L}\right)$, we have $\Gamma_{{ }^{c} T_{0}} \cap \Gamma_{{ }^{c} T_{k}} \subseteq \Gamma_{T_{0}} \cap \Gamma_{T_{k}}=\Gamma_{L}=\Gamma_{F}$. Hence, by [MW, Cor. 3.12],

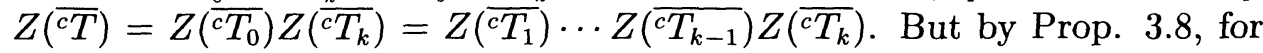
$1 \leq i \leq k$,

$$
Z\left(\overline{c T_{i}}\right) \subseteq \bar{F}\left(N_{\bar{L} / \bar{F}}(\bar{L})^{1 / d_{i}}\right), \quad d_{i}=\operatorname{gcd}\left(n_{i},[L: F]\right)
$$

Therefore, as $n_{i} \mid n$,

$$
Z(\bar{c} \bar{T}) \subseteq \bar{F}\left(N_{\bar{L} / \bar{F}}(\bar{L})^{1 / d}\right), \quad d=\operatorname{gcd}(n,[L: F]) .
$$

\section{Tame division algebras.}

We will show in Theorems 4.4-4.6 below that $\Gamma_{{ }^{c} D} \subseteq \Gamma_{D}$ for $D \in \mathcal{D}_{t}(L)$, without any restriction on roots of unity in $F$, and that for $D \in \mathcal{D}_{t}(L)$, $Z(\bar{c} \bar{D}) \subseteq \mathcal{N}(Z(\bar{D}) / \bar{F})^{1 / k}$, where $k \geq 1$ is some integer dividing $\exp \left(\operatorname{ker} \theta_{D}\right)$ which depends on which roots of unity lie in $F$. We first give some propositions which will be used in the proofs of the theorems.

Proposition 4.1. Let $n=p^{k}$ with $p$ an odd prime, $k \geq 1$, and let $\omega_{k}$ be a primitive $n$-th root of unity in L. Suppose $a, b \in F^{*}$ and $T=(a, b ; L)_{n} \in$ $\mathcal{D}_{t t r}(L)$. Then

(a) if $\mu_{p} \subseteq F$, then ${ }^{c} T \sim\left(a, b^{\ell} ; F\right)_{n / r}$ in $\mathrm{Br}(F)$, where $\ell=\left[L: F\left(\omega_{k}\right)\right]$, and $r=\left[F\left(\omega_{k}\right): F\right]$. Hence, ${ }^{c} T \in \mathcal{D}_{t t r}(F)$ and $\Gamma_{c T}=[L: F] \Gamma_{T}+\Gamma_{F}$;

(b) if $\mu_{p} \nsubseteq F$, then ${ }^{c} T=F$.

Proof. (a) For $1 \leq i \leq k$, set $\omega_{\imath}=\left(\omega_{k}\right)^{p^{k-i}}$, a primitive $p^{i}$-th root of unity. Let $F_{i}=F\left(\omega_{i}\right)$. Let $t$ be maximal with $\omega_{t} \in F$ so that $F=F_{t}$ and $\left[F_{i+1}: F_{i}\right]=p$ for $i \geq t$. (Note that $r=\left[F_{k}: F\right]=\left[F_{k}: F_{t}\right]=p^{k-t}$, so $p^{t}=n / r$.) Then by [M, \#15, p. 144],

$$
\operatorname{cor}_{F_{2+1} / F_{2}}\left(\left(a, b, \omega_{i+1} ; F_{i+1}\right)_{p^{i+1}}\right) \sim\left(\left(a, b, \omega_{i} ; F_{i}\right)_{p^{i}}\right) .
$$


So it follows that

$$
\operatorname{cor}_{F_{k} / F}\left(\left(a, b, ; F_{k}\right)_{n}\right) \sim\left(a, b ; F_{t}\right)_{p^{t}}=(a, b ; F)_{n / r} .
$$

Hence, by Prop. 1.4,

$$
\begin{aligned}
{ }^{c} T & \sim \operatorname{cor}_{L / F}\left((a, b, ; L)_{n}\right) \sim \operatorname{cor}_{F_{k} / F}\left(\operatorname{cor}_{L / F}\left((a, b, ; L)_{n}\right)\right) \\
& \sim \operatorname{cor}_{F_{k} / F}\left(\left(a, b^{\ell} ; F_{k}\right)_{n}\right) \sim\left(a, b^{\ell} ; F_{k}\right)_{n / r} .
\end{aligned}
$$

The second statement follows easily by Prop. 3.1.

(b) Let $K=F\left(\omega_{1}\right)$ and let $s$ be maximal with $\omega_{s} \in K$ so that $K=F_{s}$ and $\left[F_{i+1}: F_{i}\right]=p$ for $i \geq s$. As above, it follows by [M, \#15, p. 144] that

$$
\operatorname{cor}_{F_{k} / K}\left(\left(a, b, \omega_{k} ; F_{k}\right)_{p^{k}}\right) \sim\left(a, b, \omega_{s} ; K\right)_{p^{s}} .
$$

If $\operatorname{Gal}(K / F)=\langle\sigma\rangle$, then $\sigma\left(\omega_{s}\right)=\omega_{s}^{u}$ where $u^{m} \equiv 1\left(\bmod p^{s}\right)$ for $m=$ $[K: F]$ and $u \not \equiv 1(\bmod p)$ since $\sigma$ moves $\omega_{1}$. It follows easily that $N_{K / F}\left(\omega_{s}\right)=1$ and so $\operatorname{cor}_{K / F}\left(\left(a, b, \omega_{s} ; K\right)_{p^{s}}\right) \sim F$ by [M, \#16, p. 145]. So $\operatorname{cor}_{F_{k} / F}\left(\left(a, b, \omega_{k} ; F_{k}\right)_{n}\right) \sim F$. Hence by Prop. 1.4., as $N_{L / F_{k}}(b)=b^{\ell}, \ell=[L$ : $\left.F_{k}\right]$,

$$
\begin{aligned}
{ }^{c} T \sim \operatorname{cor}_{L / F}\left((a, b ; L)_{n}\right) \sim \operatorname{cor}_{F_{k} / F}\left(\operatorname{cor}_{L / F_{k}}\left((a, b ; L)_{n}\right)\right) & \sim \\
& \sim \operatorname{cor}_{F_{k} / F}\left(\left(a, b^{\ell} ; F_{k}\right)_{n}\right) \sim F .
\end{aligned}
$$

Proposition 4.2. Let $n=2^{k}, k \geq 3$, and let $\omega_{k}$ be a primitive $n$-th root of unity in L. Suppose $a, b \in F^{*}$ and $T=(a, b ; L)_{n} \in \mathcal{D}_{\text {ttr }}(L)$. Then

(a) if $\mu_{4} \subseteq F$, then ${ }^{c} T \sim\left(a, b^{\ell} ; F\right)_{n / r} \otimes_{F}\left(a, b^{\ell} ; F\right)_{2} \sim\left(a, b^{\ell e} ; F\right)_{n / r}$ in $\operatorname{Br}(F)$, where $\ell=\left[L: F\left(\omega_{k}\right)\right], r=\left[F\left(\omega_{k}\right): F\right]$ and $e=1+\frac{n}{2 r}$ is an odd integer. Hence ${ }^{c} T \in \mathcal{D}_{\text {trr }}(F)$ and ${ }^{c} T=[L: F] \Gamma_{T}+\Gamma_{F}$;

(b) if $\mu_{4} \nsubseteq F$, then ${ }^{c} T=(a, b ; F)_{2}$ or $=F$, and ${ }^{c} T \in \mathcal{D}_{t t r}(F)$.

Proof. (a) For $1 \leq i \leq k$, set $\omega_{i}=\left(\omega_{k}\right)^{2^{k-i}}$, a primitive $2^{i}$-th root of unity. Let $F_{i}=F\left(\omega_{i}\right)$. Let $t$ be maximal with $\omega_{t} \in F$ so that $F=F_{t}$ and $\left[F_{i+1}: F_{i}\right]=2$ for $i \geq t$. (Note that $t \geq 2$ as $\mu_{4} \subseteq F$.) Then by [M, \#15 and \#13, p. 144],

$$
\begin{aligned}
\operatorname{cor}_{F_{i+1} / F_{i}}\left(\left(a, b, \omega_{i+1} ; F_{i+1}\right)_{2^{i+1}}\right) & \sim\left(\left(a, b,-\omega_{i} ; F_{i}\right)_{2^{i}}\right) \\
& \sim\left(\left(a, b, \omega_{i} ; F_{i}\right)_{2^{i}}\right) \otimes_{F}\left(\left(a, b,-1 ; F_{i}\right)_{2}\right) .
\end{aligned}
$$

Since $\operatorname{cor}_{F_{i} / F_{i-1}}\left(\left(a, b,-1 ; F_{i}\right)_{2}\right) \sim F_{i-1}$ by Prop. 1.4 as $N_{F_{i} / F_{i-1}}(b)=b^{2}$, we end up with $\operatorname{cor}_{F_{k} / F}\left(\left(a, b ; F_{k}\right)_{n}\right) \sim(a, b ; F)_{n / r} \otimes_{F}(a, b ; F)_{2}$. Hence, by Prop. 1.4 ,

$$
\begin{aligned}
{ }^{c} T & \sim \operatorname{cor}_{L / F}\left((a, b, ; L)_{n}\right) \sim \operatorname{cor}_{F_{k} / F}\left(\operatorname{cor}_{L / F_{k}}\left((a, b, ; L)_{n}\right)\right) \\
& \sim \operatorname{cor}_{F_{k} / F}\left(\left(a, b^{\ell} ; F_{k}\right)_{n}\right) \sim\left(a, b^{\ell} ; F_{k}\right)_{n / r} \otimes\left(a, b^{\ell} ; F\right)_{2} .
\end{aligned}
$$


The second statement follows easily by Prop. 3.1.

(b) Let $K=F_{2}=F(\sqrt{-1})$ and let $s$ be maximal with $\omega_{s} \in K$ so that $K=F_{s}$ and $\left[F_{i+1}: F_{i}\right]=2$ for $i \geq s$. As above, it follows by [M, \#15 and \#13, p. 144] that

$$
\operatorname{cor}_{F_{k} / K}\left(\left(a, b, \omega_{k} ; F_{k}\right)_{2^{k}}\right) \sim\left(a, b, \omega_{s} ; K\right)_{2^{s}} \otimes_{K}(a, b,-1 ; K)_{2} .
$$

Note that $\operatorname{cor}_{K / F}\left((a, b,-1 ; K)_{2}\right) \sim F$ by Prop. 1.4. If $\operatorname{Gal}(K / F)=\langle\sigma\rangle$, then $\sigma\left(\omega_{s}\right)=\omega_{s}^{u}$ where $u^{2} \equiv 1\left(\bmod 2^{s}\right)$ and $u \not \equiv 1(\bmod 4)$ since $\sigma$ moves $\sqrt{-1}$. It follows easily that $N_{K / F}\left(\omega_{s}\right)=1$ or -1 , and so $\operatorname{cor}_{K / F}\left(\left(a, b, \omega_{s} ; K\right)_{2^{s}}\right) \sim F$ or $(a, b,-1 ; F)_{2}$ by $\left[\mathbf{M}, \# 16\right.$ and \#7, pp. 143-145]. So $\operatorname{cor}_{F_{k} / F}\left(\left(a, b, \omega_{k} ; F_{k}\right)_{n}\right)$ $\sim F$ or $(a, b,-1 ; F)_{2}$. Hence,

$$
{ }^{c} T \sim \operatorname{cor}_{L / F}\left((a, b ; L)_{n}\right) \sim F \quad \text { or } \quad(a, b ; F)_{2} .
$$

Lemma 4.3. Let $\omega_{4}$ be a primitive fourth root of unity in $L$. Let $a, b, c, d \in$ $F^{*}$, and let $T=(a, b ; L)_{4}$ and $T_{0}=(a, b ; F)_{2}$. Suppose $T, T_{0} \in \mathcal{D}_{t t r}(L)$. Then

(a) if $\omega_{4} \in F$, then ${ }^{c} T \sim\left(a, b^{\ell} ; F\right)_{4}$ in $\mathrm{Br}(F)$, where $\ell=[L: F]$. So ${ }^{c} T \in \mathcal{D}_{t t r}(F)$, and $\Gamma_{c_{T}}=[L: F] \Gamma_{T}+\Gamma_{F}$. Also, ${ }^{c} T_{0} \sim\left(c, d^{\ell} ; F\right)$ in $\operatorname{Br}(F)$, so ${ }^{c} T_{0} \in \mathcal{D}_{t t r}(F)$, and $\Gamma_{c} T_{0}=[L: F] \Gamma_{T_{0}}+\Gamma_{F}$;

(b) if $\omega_{4} \notin F$, then ${ }^{c} T=F$.

Proof. (a) follows easily by Prop. 1.4 and Prop. 3.1. (b) can be proved by the exact same arguments in the proof of Prop. 4.2 (b).

Recall that we are assuming $L / F$ is a finite separable inertial extension of Henselian fields.

Theorem 4.4. Let $T \in \mathcal{D}_{t t r}(L)$ and $\exp \left(\Gamma_{T} / \Gamma_{L}\right)=p_{0}^{e_{0}} p_{1}^{e_{1}} \cdots p_{r}^{e_{r}}$, where $p_{0}=2, e_{0} \geq 0$, and for $1 \leq i \leq r$, the $p_{i}$ are distinct odd primes, and $e_{i}>0$. Let $T=\bigotimes_{i=0}^{r} T_{i}$ be the primary decomposition of $T$ where $T_{i}$ is the $p_{i}$ primary component of $T$. (So $\Gamma_{T}=\sum_{i=0}^{r} \Gamma_{T_{i}}$ and $\Gamma_{T_{i}} / \Gamma_{L}$ is the $p_{i}$-primary component of $\Gamma_{T} / \Gamma_{L}$.) Suppose $\mu_{p_{i}} \subseteq F$ for $1 \leq i \leq r_{0}$ and $\mu_{p_{i}} \nsubseteq F$ for $r_{0}+1 \leq i \leq r$. Then

(a) if $4 \nmid \exp \left(\Gamma_{T} / \Gamma_{L}\right)$ or $\mu_{4} \subseteq F$, then

$$
[L: F]\left(\Gamma_{T_{0}}+\Gamma_{T_{1}}+\cdots+\Gamma_{T_{r_{0}}}\right)+\Gamma_{F} \subseteq \Gamma_{c_{T}} \subseteq \Gamma_{T}, \text { and }
$$

(b) if $4 \mid \exp \left(\Gamma_{T} / \Gamma_{L}\right)$ and $\mu_{4} \nsubseteq F$, then

$$
[L: F]\left(\Gamma_{T_{1}}+\cdots+\Gamma_{T_{r_{0}}}\right)+\Gamma_{F} \subseteq \Gamma_{c T} \subseteq \Gamma_{T} .
$$


Proof. As $T_{i} \in \mathcal{D}_{t t r}(L)$ by Prop. 1.6, $T_{i}=\bigotimes_{k=1}^{\ell_{i}}\left(\alpha_{k}, \beta_{k} ; L\right)_{m_{k}}$ for some $\alpha_{k}, \beta_{k} \in$ $L^{*}$, and some $m_{k}$ powers of $p_{i}$ for $1 \leq k \leq \ell_{i}\left(\right.$ so $\Gamma_{T_{i}} / \Gamma_{L}=\underset{k=1}{\oplus}\left\langle\frac{1}{m_{k}} v\left(\alpha_{k}\right)+\Gamma_{L}\right.$, $\left.\left.\frac{1}{m_{k}} v\left(\beta_{k}\right)+\Gamma_{L}\right\rangle\right)$. Since $\Gamma_{L}=\Gamma_{F}, T_{i} \sim\left(\bigotimes_{k=1}^{\ell_{i}}\left(a_{k}, b_{k} ; L\right)_{m_{k}}\right) \otimes_{F} S_{i}$ for some $S_{i} \in \mathcal{D}_{i s}(L)$ and $a_{k}, b_{k} \in F^{*}$ with $v\left(a_{k}\right)=v\left(\alpha_{k}\right), v\left(b_{k}\right)=v\left(\beta_{k}\right)\left(1 \leq k \leq \ell_{i}\right)$. Let $T_{i}^{\prime} \sim{\stackrel{\ell}{\ell_{i}}}_{k=1}\left(a_{k}, b_{k} ; L\right)_{m_{k}}$. As $T_{i} \in \mathcal{D}_{t t r}(L)$, Prop. 3.1 shows $T_{i}^{\prime} \in \mathcal{D}_{t t r}(L)$, and $\Gamma_{T_{i}^{\prime}}=\Gamma_{T_{i}}$. Since $T_{i} \sim T_{i}^{\prime} \otimes_{L} S_{i}$ where $T_{i}^{\prime} \in \mathcal{D}_{t t r}(L)$, and $S_{i} \in \mathcal{D}_{i s}(L)$, by Prop. 1.7, $\Gamma_{T_{i}}=\Gamma_{T_{i}^{\prime}}+\Gamma_{S_{i}}$. Also, as $L / F$ is inertial, ${ }^{c} S_{i} \in \mathcal{D}_{i s}(F)$ and $\Gamma_{{ } S_{i}} \subseteq \Gamma_{S_{i}}$ by Theorem 2.4 .

For $1 \leq i \leq r_{0}$, as $\mu_{p_{i}} \subseteq F$, by Prop. $4.1^{c} T_{i}^{\prime} \sim{\stackrel{\bigotimes}{\ell_{i}}}_{k=1}\left(a_{k}, b_{k}^{n_{k}} ; F\right)_{m_{k} / r_{k}}$ where $n_{k}=\left[L: F\left(\omega_{m_{k}}\right)\right]\left(\omega_{m_{k}}\right.$, a primitive $m_{k}$-th root of unity) and $r_{k}=$ $\left[F\left(\omega_{m_{k}}\right): F\right]$. Let $A_{k}$ be the underlying division algebra of $\left(a_{k}, b_{k}^{n_{k}} ; F\right)_{m_{k} / r_{k}}$. As $\left(a_{k}, b_{k} ; F\right)_{m_{k} / r_{k}} \in \mathcal{D}_{t t r}(F)$, by Prop. 3.1, $A_{k} \in \mathcal{D}_{t t r}(F)$ and

$$
\begin{aligned}
\Gamma_{A_{k}} / \Gamma_{F} & =n_{k}\left(\left\langle\frac{r_{k}}{m_{k}} v\left(a_{k}\right)+\Gamma_{F}, \frac{r_{k}}{m_{k}} v\left(b_{k}\right)+\Gamma_{F}\right\rangle\right) \\
& =[L: F]\left(\left\langle\frac{1}{m_{k}} v\left(a_{k}\right)+\Gamma_{F}, \frac{1}{m_{k}} v\left(b_{k}\right)+\Gamma_{F}\right\rangle\right)
\end{aligned}
$$

as $n_{k} r_{k}=[L: F]$. Then, by Prop. 3.1 again, $A:={\underset{k=1}{\ell_{i}}}_{k=1} A_{k} \in \mathcal{D}_{t t r}(F)$ and $\Gamma_{A} / \Gamma_{F}={\underset{k=1}{\ell_{i}}}_{\oplus^{\prime}}\left(\Gamma_{A_{k}} / \Gamma_{F}\right)=[L: F]\left(\Gamma_{T_{i}^{\prime}} / \Gamma_{F}\right)$. So, ${ }^{c} T_{i}^{\prime}=A \in \mathcal{D}_{t t r}(F)$ and $\Gamma_{c} T_{i}^{\prime} / \Gamma_{F}=[L: F]\left(\Gamma_{T_{i}^{\prime}} / \Gamma_{F}\right)=[L: F]\left(\Gamma_{T_{i}} / \Gamma_{F}\right)$. Since ${ }^{c} T_{i} \sim{ }^{c} T_{i}^{\prime} \otimes_{F}$ ${ }^{c} S_{i}, \Gamma_{{ }^{c} T_{i}}=\Gamma_{{ }^{c} T_{i}^{\prime}}+\Gamma_{c} S_{i}$ by Prop. 1.7. So,

$$
[L: F] \Gamma_{T_{i}}+\Gamma_{F} \subseteq \Gamma_{c T_{i}}=\Gamma_{c T_{i}^{\prime}}+\Gamma_{c S_{i}} \subseteq \Gamma_{T_{i}^{\prime}}+\Gamma_{S_{i}}=\Gamma_{T_{i}}
$$

For $r_{0}+1 \leq i \leq r$, as $\mu_{p_{i}} \nsubseteq F$ by assumption, ${ }^{c} T_{i}^{\prime}=F$ by Prop. 4.1. So ${ }^{c} T_{i}={ }^{c} S_{i}$, and $\Gamma^{c} T_{i}=\Gamma_{c} S_{i} \subseteq \Gamma_{S_{i}} \subseteq \Gamma_{T_{i}}$.

(a) If $4 \nmid \exp \left(\Gamma_{T} / \Gamma_{L}\right)$ or $\mu_{4} \subseteq F$, then the same argument proving of the case when $1 \leq i \leq r_{0}$ gives us $[L: F] \Gamma_{T_{0}}+\Gamma_{F} \subseteq \Gamma_{{ }^{c} T_{0}} \subseteq \Gamma_{T_{0}}$, using Prop. 4.2 and Lemma 4.3 instead of Prop. 4.1. Thus, since $\Gamma_{T}=\sum_{i=0}^{r} \Gamma_{T_{i}}$ and $\Gamma_{{ } T}=\sum_{i=0}^{r} \Gamma_{{ }^{\prime} T_{i}}$ by [JW2, Cor. 1.15], $[L: F]\left(\Gamma_{T_{0}}+\Gamma_{T_{1}}+\cdots+\Gamma_{T_{r_{0}}}\right)+\Gamma_{F} \subseteq$ $\Gamma_{c T} \subseteq \Gamma_{T}$.

(b) If $4 \mid \exp \left(\Gamma_{T} / \Gamma_{L}\right)$ and $\mu_{4} \nsubseteq F$, then by Prop. 4.2 and Lemma 4.3, ${ }^{c} T_{0}^{\prime} \sim{\stackrel{\ell_{0}}{\otimes}}^{A_{k}} A_{k}$, where for $1 \leq k \leq \ell_{0}, A_{k}=\left(a_{k}, b_{k} ; F\right)_{2}$ or $=F$. Hence ${ }^{c} T_{0}^{\prime} \in \stackrel{\mathcal{D}}{t t r}^{k=1}(F)$ and $\Gamma^{c} T_{0}^{\prime} \subseteq \Gamma_{T_{0}^{\prime}}$. As ${ }^{c} T_{0} \sim{ }^{c} T_{0}^{\prime} \otimes{ }^{c} S_{0}$ with ${ }^{c} S_{0} \in \mathcal{D}_{i s}(F)$, by Prop. 1.7, $\Gamma_{c T_{0}}=\Gamma_{c T_{0}^{\prime}}+\Gamma_{c S_{0}} \subseteq \Gamma_{T_{0}^{\prime}}+\Gamma_{S_{0}}=\Gamma_{T_{0}}$. Therefore, $[L$ : $F]\left(\Gamma_{T_{1}}+\cdots+\Gamma_{T_{r_{0}}}\right)+\Gamma_{F} \subseteq \Gamma_{c_{T}} \subseteq \Gamma_{T}$. 
Theorem 4.5. If $D \in \mathcal{D}_{t}(L)$, then $\Gamma_{{ } D} \subseteq \Gamma_{D}$.

Proof. By Prop. 1.7, there are $S \in \mathcal{D}_{i s}(L)$ and $T \in \mathcal{D}_{t t r}(L)$ such that $D \sim S \otimes_{L} T$ in $\operatorname{Br}(L)$, whence $\Gamma_{D}=\Gamma_{S}+\Gamma_{T}$. Then by Theorem 2.4 and Theorem 4.4, $\Gamma_{{ } S} \subseteq \Gamma_{S}$ and $\Gamma_{{ }_{c} T} \subseteq \Gamma_{T}$. Also, by Theorem 2.4, ${ }^{c} S$ and ${ }^{c} T$ are tame over $F$. As ${ }^{c} D \sim{ }^{c} S \otimes_{F}{ }^{c} T$ in $\operatorname{Br}(F)$, by [JW2, Cor. 6.7], $\Gamma_{c_{D}} \subseteq \Gamma_{c_{S}}+\Gamma_{c_{T}} \subseteq \Gamma_{S}+\Gamma_{T}=\Gamma_{D}$.

Now, we will give relations between $Z(\bar{D})$ and $Z(\bar{c} \bar{D})$ and between $\exp \left(\operatorname{ker} \theta_{D}\right)$ and $\exp \left(\operatorname{ker} \theta_{c_{D}}\right)$ for $D \in \mathcal{D}_{t}(L)$.

Theorem 4.6. Let $D \in \mathcal{D}_{t}(L)$ and $t=\exp \left(\operatorname{ker} \theta_{D}\right)$. Let $t=2^{e_{0}} p_{1}^{e_{1}} \cdots p_{r}^{e_{r}}$, where $e_{0} \geq 0$, and the $p_{i}$ are distinct odd primes and $e_{i}>0$ for $1 \leq i \leq r$. Suppose $\mu_{p_{i}} \subseteq F$ for $1 \leq i \leq r_{0}$, and $\mu_{p_{i}} \nsubseteq F$ for $r_{0}+1 \leq i \leq r$. Let $s=2^{e_{0}} p_{1}^{e_{1}} \cdots p_{r_{0}}^{e_{r_{0}}}$ and $s^{\prime}=p_{1}^{e_{1}} \cdots p_{r_{0}}^{e_{r_{0}}}$. Then

(a) if $4 \nmid t$ or $\mu_{4} \subseteq F$, then $\exp \left(\operatorname{ker} \theta_{c_{D}}\right)=s / \operatorname{gcd}([L: F], s)$ and $Z(\bar{c} \bar{D}) \subseteq$ $\mathcal{N}(Z(\bar{D}) / \bar{F})^{1 / n}$ where $n=\operatorname{gcd}([L: F], s) \cdot(t / s)$, and

(b) if $4 \mid t$ and $\mu_{4} \nsubseteq F$, then $\exp \left(\operatorname{ker} \theta_{c_{D}}\right)=\varepsilon s^{\prime} / \operatorname{gcd}\left([L: F], s^{\prime}\right)$ where $\varepsilon=1$ or 2 , and $Z\left(\overline{{ }^{c} D}\right) \subseteq \mathcal{N}(Z(\bar{D}) / \bar{F})^{1 / n^{\prime}}$ where $n^{\prime}=\operatorname{gcd}\left([L: F], s^{\prime}\right) \cdot\left(t / s^{\prime}\right)$.

Proof. By Prop. 1.7, there is a noncanonical decomposition in $\operatorname{Br}(L), D \sim$ $S^{\prime} \otimes_{L} T^{\prime}$ where $S^{\prime} \in \mathcal{D}_{i s}(L)$ and $T^{\prime} \in \mathcal{D}_{t t r}(L)$. As $\operatorname{ker}\left(\theta_{D}\right)=\Gamma_{T^{\prime}} / \Gamma_{L}$, we have $t=\exp \left(\operatorname{ker} \theta_{D}\right)=\exp \left(\Gamma_{T^{\prime}} / \Gamma_{L}\right)$. Let $T^{\prime}=\bigotimes_{i=0}^{r} T_{i}^{\prime}$ be the primary decomposition of $T^{\prime}$. Since $T_{i}^{\prime} \in \mathcal{D}_{t t r}(L)$, by Prop. 1.6, $T_{i}^{\prime}$ is a tensor product of tame totally ramified symbol algebras

$$
T_{i}^{\prime}=\bigotimes_{j=1}^{j(i)}\left(x_{(i, 2 j-1)}, x_{(i, 2 j)} ; L\right)_{t(i, j)}
$$

where

$$
\Gamma_{T_{2}^{\prime}} / \Gamma_{L}=\stackrel{\oplus}{j=1}_{j(i)}\left|\left\langle\frac{1}{t(i, j)} v\left(x_{(i, 2 j-1)}\right)+\Gamma_{L}, \frac{1}{t(i, j)} v\left(x_{(i, 2 j)}\right)+\Gamma_{L}\right\rangle\right|
$$

(cf. Prop. 3.1). Note that as

$$
\exp \left(\Gamma_{T_{i}^{\prime}} / \Gamma_{L}\right)=\text { the } p_{i} \text {-component of } \exp \left(\Gamma_{T^{\prime}} / \Gamma_{L}\right)=p_{i}^{e_{i}},
$$

each $t(i, j) \mid p_{i}^{e_{i}}$. (For $n \in \mathbb{Z}$ and $p$ a prime, if we write $n=n_{p} \cdot n_{p}^{\prime}$ where $n_{p}$ is a power of $p$ and $n_{p}^{\prime}$ is prime to $p$, then we refer to $n_{p}$ as the $p$-component of $n$.) 
Since $\Gamma_{L}=\Gamma_{F}, x_{\left(i, j^{\prime}\right)}=u_{\left(i, j^{\prime}\right)} y_{\left(i, j^{\prime}\right)}, 1 \leq j^{\prime} \leq 2 j(i)$, for some $y_{\left(i, j^{\prime}\right)} \in F^{*}$ and $u_{\left(i, j^{\prime}\right)} v$-units in $L^{*}$. Note that

$$
\begin{aligned}
& \left(x_{(i, 2 j-1)}, x_{(i, 2 j)} ; L\right)_{t(i, j)} \\
\sim & \left(u_{(i, 2 j-1)}, x_{(i, 2 j)} ; L\right)_{t(i, j)} \otimes\left(y_{(i, 2 j-1)}, u_{(i, 2 j)} ; L\right)_{t(i, j)} \otimes\left(y_{(i, 2 j-1)}, y_{(i, 2 j)} ; L\right)_{t(i, j)},
\end{aligned}
$$

and

$$
\left[\left(u_{(i, 2 j-1)}, x_{(i, 2 j)} ; L\right)_{t(i, j)} \otimes\left(y_{(i, 2 j-1)}, u_{(i, 2 j)} ; L\right)_{t(i, j)}\right] \in \operatorname{Br}\left(L_{n r} / L\right) .
$$

Let $S_{i}$ be the underlying division algebra of

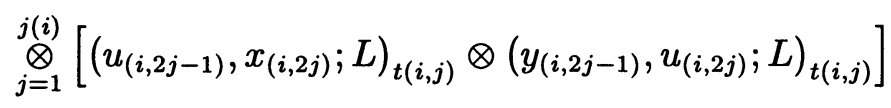

and $T_{i}=\otimes_{j=1}^{j(i)}\left(y_{(i, 2 j-1)}, y_{(i, 2 j)} ; L\right)_{t(i, j)}$. It follows from Prop. 3.1, that $T:=$ $\stackrel{\otimes}{r}_{i=0}^{\otimes} T_{i} \in \mathcal{D}_{t t r}(L)$. Then by replacing $S^{\prime}$ and $T^{\prime}$.with the underlying division algebra of $S^{\prime} \otimes\left(\otimes_{i=0}^{r} S_{i}\right)$ and $T$, we have in $\operatorname{Br}(L), D \sim S \otimes_{L} T$ where $S \in \mathcal{D}_{i s}(L), T=\stackrel{\otimes}{i=0}_{i} T_{i}$ and $T_{i}=\bigotimes_{j=1}^{j(i)}\left(y_{(i, 2 j-1)}, y_{(i, 2 j)} ; L\right)_{t(i, j)}$ with $y_{\left(i, j^{\prime}\right)} \in F^{*}$. And ${ }^{c} D \sim{ }^{c} S \otimes_{F}{ }^{c} T$ where ${ }^{c} S \in \mathcal{D}_{i s}(F)$ by Theorem 2.4.

(a) Suppose $4 \nmid t$ or $\mu_{4} \subseteq F$.

By Prop. 4.1 (a) for $1 \leq i \leq r_{0}$, and by Prop. 4.2 (a) and Lemma

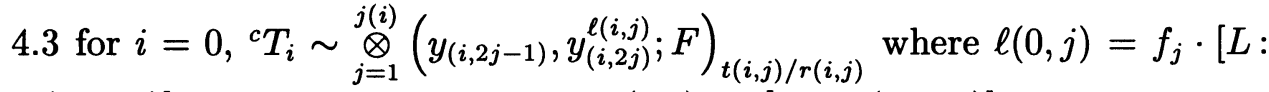
$\left.F\left(\omega_{t(0, j)}\right)\right]$ with $f_{j}$ an odd integer, $\ell(i, j)=\left[L: F\left(\omega_{t(i, j)}\right)\right]$ for $1 \leq i \leq r_{0}$ and $r(i, j)=\left[F\left(\omega_{t(i, j)}\right): F\right]$ for $0 \leq i \leq r_{0}\left(\omega_{t(i, j)}\right.$ is a primitive $t(i, j)$ th root of unity). Let $d(i, j)=\operatorname{gcd}\left(\ell(i, j), \frac{t(i, j)}{r(i, j)}\right), \ell^{\prime}(i, j)=\frac{\ell(i, j)}{d(i, j)}$, and $t^{\prime}(i, j)=\frac{t(i, j)}{r(i, j) d(i, j)}=\frac{t(i, j)}{\operatorname{gcd}([L: F], t(i, j))}$ so that $\operatorname{gcd}\left(\ell^{\prime}(i, j), t^{\prime}(i, j)\right)=1$. Then $r(i, j) d(i, j)=\operatorname{gcd}([L: F], t(i, j))$ as $r(i, j) \ell(i, j)=[L: F]$ for $1 \leq i \leq r_{0}$ and $r(0, j) \ell(0, j)=[L: F] f_{j}, 2 \nmid f_{j}$, and $t(0, j)$ are powers of 2 . Then ${ }^{c} T_{i} \sim \stackrel{\otimes}{j=1}^{j(i)}\left(y_{(i, 2 j-1)}, y_{(i, 2 j)}^{\ell^{\prime}(i, j)} ; F\right)_{t^{\prime}(i, j)}$.

For $r_{0}+1 \leq i \leq r$, as $\mu_{p_{i}} \nsubseteq F,{ }^{c} T_{i}=F$ by Prop. 4.1. So ${ }^{c} T \sim{\underset{i=0}{r_{0}}}^{c} T_{i}$ in $\operatorname{Br}(F)$. Since

$$
\begin{aligned}
\Gamma_{T} / \Gamma_{L}=\Gamma_{T} / \Gamma_{F} & =\bigoplus_{i=0}^{r}\left(\Gamma_{T_{i}} / \Gamma_{F}\right)= \\
& =\bigoplus_{i=0}^{r} \bigoplus_{j=1}^{j(i)}\left\langle\frac{1}{t(i, j)} v\left(y_{(i, 2 j-1)}\right)+\Gamma_{F}, \frac{1}{t(i, j)} v\left(y_{(i, 2 j)}\right)+\Gamma_{F}\right\rangle
\end{aligned}
$$


and $\Gamma_{c T_{i}} / \Gamma_{F} \subseteq \Gamma_{T_{i}} / \Gamma_{F}$, it follows from Prop. 2.4 that ${ }^{c} T \cong{\stackrel{\otimes}{r_{0}}}^{c}{ }^{c} T_{i} \in$ $\mathcal{D}_{t t r}(F)$ and

$$
\begin{aligned}
\Gamma_{c T} / \Gamma_{F}=\bigoplus_{i=0}^{r_{0}} & \left(\Gamma_{{ } T_{i}} / \Gamma_{F}\right)= \\
& =\bigoplus_{i=0}^{r_{0}} \bigoplus_{j=1}^{j(i)}\left\langle\frac{1}{t^{\prime}(i, j)} v\left(y_{(i, 2 j-1)}\right)+\Gamma_{F}, \frac{1}{t^{\prime}(i, j)} v\left(y_{(i, 2 j)}\right)+\Gamma_{F}\right\rangle,
\end{aligned}
$$

as $\operatorname{gcd}\left(\ell^{\prime}(i, j), t^{\prime}(i, j)\right)=1$.

Let $T^{\prime}=\bigotimes_{i=0}^{r_{0}} T_{i}$. Then

$$
\begin{aligned}
\Gamma_{T^{\prime}} / \Gamma_{L} & =\bigoplus_{i=0}^{r_{0}}\left(\Gamma_{T_{i}} / \Gamma_{L}\right) \\
& =\bigoplus_{i=0 j=1}^{r_{0}} \bigoplus_{j(i)}\left\langle\frac{1}{t(i, j)} v\left(y_{(i, 2 j-1)}\right)+\Gamma_{L}, \frac{1}{t(i, j)} v\left(y_{(i, 2 j)}\right)+\Gamma_{L}\right\rangle .
\end{aligned}
$$

Since $\Gamma_{L}=\Gamma_{F}$ and

$$
\frac{t^{\prime}(i, j)=t(i, j)}{\operatorname{gcd}([L: F], t(i, j)), \Gamma_{c_{T}} / \Gamma_{F}=[L: F]\left(\Gamma_{T^{\prime}} / \Gamma_{L}\right)} .
$$

Recall that $s=2^{e_{0}} p_{1}^{e_{1}} \cdots p_{r_{0}}^{e_{r_{0}}}=\exp \left(\Gamma_{T^{\prime}} / \Gamma_{L}\right)$, as $t=2^{e_{0}} p_{1}^{e_{1}} \cdots p_{r}^{e_{r}}=$ $\exp \left(\Gamma_{T} / \Gamma_{L}\right)$. Note that $(t / s) \Gamma_{T} \subseteq \Gamma_{T^{\prime}}$, as $\exp \left(\Gamma_{T_{i}} / \Gamma_{L}\right) \mid(t / s)$ for $i>r_{0}$. Also, for $0 \leq i \leq r_{0}$, as each $t(i, j) \mid s$, each $t(i, j) / t^{\prime}(i, j)=\operatorname{gcd}([L: F]$, $t(i, j)) \mid \operatorname{gcd}([L: F], s)$. Therefore, $\operatorname{gcd}([L: F], s) \Gamma_{T^{\prime}} \subseteq \Gamma_{c_{T}}$. Hence, as $n=\operatorname{gcd}([L: F], s) \cdot(t / s)$, we have $n \Gamma_{T} \subseteq \Gamma_{c_{T}}$.

Note that if $A$ is a finite abelian group (written additively) and $\ell$ is a positive integer, then

$$
\exp (\ell \cdot A)=\frac{\exp (A)}{\operatorname{gcd}(\ell, \exp (A))}
$$

(This follows from the fact that there is $b \in A$ with $o(b)=\exp (A)$ and the formula $o(\ell \cdot b)=o(b) / \operatorname{gcd}(\ell, o(b))$.) Since $\Gamma_{c_{T}} / \Gamma_{F}=[L: F]\left(\Gamma_{T^{\prime}} / \Gamma_{L}\right)$ and $\Gamma_{T^{\prime}} / \Gamma_{L}$ is a finite abelian group,

$$
\exp \left(\Gamma_{c_{T}} / \Gamma_{F}\right)=\frac{\exp \left(\Gamma_{T^{\prime}} / \Gamma_{L}\right)}{\operatorname{gcd}\left([L: F], \exp \left(\Gamma_{T^{\prime}} / \Gamma_{L}\right)\right)}=s / \operatorname{gcd}([L: F], s)
$$

by (4.7) above. Since ${ }^{c} D \sim{ }^{c} S \otimes_{F}{ }^{c} T$ with ${ }^{c} S \in \mathcal{D}_{i s}(F)$ and ${ }^{c} T \in \mathcal{D}_{t t r}(F)$, by Prop. $1.7 \exp \left(\operatorname{ker} \theta_{c_{D}}\right)=\exp \left(\Gamma_{c_{T}} / \Gamma_{F}\right)=s / \operatorname{gcd}([L: F], s)$.

Let

$$
K=F\left(\left\{y_{(i, 2 j-1)}^{1 / t^{\prime}(i, j)}, y_{(i, 2 j)}^{1 / t^{\prime}(i, j)}\right\}_{i=0 j=1}^{r_{0} j(i)}\right)
$$


Then $K / F$ is totally ramified and $\Gamma_{K}=\Gamma_{c_{T}}$. Since ${ }^{c} S \in \mathcal{D}_{i s}(F)$ and $K / F$ is totally ramified, by [JW2, Cor. 5.13] $Z\left(\overline{\left({ }^{c} S\right)_{K}}\right)=\mathcal{F}\left(\theta_{c_{S}}\left(\left(\Gamma_{{ }^{c} S} \cap \Gamma_{K}\right) / \Gamma_{F}\right)\right)$, where $\left({ }^{c} S\right)_{K}$ is the underlying division algebra of ${ }^{c} S \otimes_{F} K$. Also since ${ }^{c} D \sim$ ${ }^{c} S \otimes_{F}{ }^{c} T$ with ${ }^{c} S \in \mathcal{D}_{i s}(F)$ and ${ }^{c} T \in \mathcal{D}_{t t r}(F)$, by Prop. $1.7 Z\left(\overline{{ }^{c} D}\right)=$ $\mathcal{F}\left(\theta_{c_{S}}\left(\left(\Gamma_{c_{S}} \cap \Gamma_{c_{T}}\right) / \Gamma_{F}\right)\right)$. Hence $Z(\bar{c} \bar{D})=Z\left(\overline{\left({ }^{c} S\right)_{K}}\right)$ as $\Gamma_{c_{T}}=\Gamma_{K}$.

Now, since $K / F$ is Galois and $K \cap L=F$ with $K / F$ totally ramified and $L / F$ inertial, $L$ and $K$ are linearly disjoint over $F$ and $L \otimes_{F} K$ is the field $L \cdot K$. So by [D1, p. 56, Ex. 1], $\left({ }^{c} S\right)_{K} \sim \operatorname{cor}_{L K / K}\left(S_{L K}\right)$ in $\operatorname{Br}(K)$, where $S_{L K}$ is the underlying division algebra of $S \otimes_{L} L K$. Since $S_{L K} \in \mathcal{D}_{i s}(L K)$ and $L K / K$ is inertial, by Theorem 2.4 ,

$$
Z\left(\overline{{ }^{c} D}\right)=Z\left(\overline{\left({ }^{c} S\right)_{K}}\right) \subseteq \mathcal{N}\left(Z\left(\overline{S_{L K}}\right) / \bar{K}\right) .
$$

Recall that $n=\operatorname{gcd}([L: F], s) \cdot(t / s)$. We showed just before (4.7) that $n \Gamma_{T} \subseteq \Gamma_{c_{T}}=\Gamma_{K}=\Gamma_{L K}$. So, $n\left(\left(\Gamma_{S} \cap \Gamma_{T}\right) /\left(\Gamma_{S} \cap \Gamma_{L K}\right)\right)=0$.

Let

$$
\theta_{S}: \Gamma_{S} / \Gamma_{L} \rightarrow \operatorname{Gal}(Z(\bar{S}) / \bar{L})
$$

be the map of (1.5). Then by [JW2, Lemma 5.1], $\theta_{S}$ is an isomorphism and $Z(\bar{S}) / \bar{L}$ is abelian Galois as $S \in \mathcal{D}_{i s}(L)$. By Prop. 1.7, $\theta_{S}\left(\left(\Gamma_{S} \cap \Gamma_{T}\right) / \Gamma_{L}\right)=$ $\operatorname{Gal}(Z(\bar{S}) / Z(\bar{D}))$. Also, since $S \in \mathcal{D}_{\imath s}(L)$ and $L K / L$ is totally ramified, by [JW2, Cor. 5.13] again $\theta_{S}\left(\left(\Gamma_{S} \cap \Gamma_{L K}\right) / \Gamma_{L}\right)=\operatorname{Gal}\left(Z(\bar{S}) / Z\left(\overline{S_{L K}}\right)\right)$. So

$$
\begin{aligned}
\operatorname{Gal}\left(Z\left(\overline{S_{L K}}\right) / Z(\bar{D})\right) & \cong \operatorname{Gal}(Z(\bar{S}) / Z(\bar{D})) / \mathrm{Gal}\left(Z(\bar{S}) / Z\left(\overline{S_{L K}}\right)\right) \\
& \cong\left(\Gamma_{S} \cap \Gamma_{T}\right) /\left(\Gamma_{S} \cap \Gamma_{L K}\right) .
\end{aligned}
$$

Hence $\operatorname{Gal}\left(Z\left(\overline{S_{L K}}\right) / Z(\bar{D})\right)$ is an abelian $n$-torsion group by the preceding paragraph. Note that $\mu_{n} \subseteq Z(\bar{D})$ as $n \mid t=\exp \left(\Gamma_{T} / \Gamma_{L}\right)$, and $\mu_{t} \subseteq \bar{L} \subseteq$ $Z(\bar{D})$. Hence by Kummer theory $Z\left(\overline{S_{L K}}\right)$ is an $n$-Kummer extension of $Z(\bar{D})$, i.e., $Z\left(\overline{S_{L K}}\right)=Z(\bar{D})\left(\left\{\alpha_{m}^{1 / n}\right\}_{m=1}^{s}\right)$ for some $\alpha_{m} \in Z(\bar{D})$. Therefore,

$$
Z\left(\overline{S_{L K}}\right) \subseteq Z(\bar{D})^{1 / n}
$$

Since $\bar{K}=\bar{F}$, by (4.8) and (4.9) above

$$
Z(\bar{D}) \subseteq \mathcal{N}\left(Z(\bar{D})^{1 / n} / \bar{F}\right) \subseteq \mathcal{N}(Z(\bar{D}) / \bar{F})^{1 / n} .
$$

(b) Suppose $4 \mid t$ and $\mu_{4} \nsubseteq F$.

By Prop. 4.1, for $1 \leq i \leq r_{0}$, as shown in proof of (a)

$$
{ }^{c} T_{i} \sim \bigotimes_{j=1}^{j(i)}\left(y_{(i, 2 j-1)}, y_{(i, 2 j)}^{\ell(i, j)} ; F\right)_{t(i, j) / r(i, j)}
$$

where $\ell(i, j)=\left[L: F\left(\omega_{t(i, j)}\right)\right]$ and $r(i, j)=\left[F\left(\omega_{t(i, j)}\right): F\right]$, and for $r_{0}+1 \leq$ $i \leq r,{ }^{c} T_{i}=F$. Also, by Prop. 4.4 and Lemma 4.3, ${ }^{c} T_{0} \sim \stackrel{\otimes}{j=1}_{j(0)}^{\otimes} A_{j}$ where 
$A_{j}=\left(y_{(0,2 j-1)}, y_{(0,2 j)} ; F\right)_{2}$ or $=F$. So we have ${ }^{c} T \sim\left(\underset{j=1}{\stackrel{\otimes}{\otimes} A_{j}}\right) \otimes_{F}\left({\stackrel{\left({ }_{0}\right.}{\otimes}}_{i=1}^{c} T_{i}\right)$. Then by the exact same arguments as in proof of (a), ${ }^{c} T \cong\left(\underset{j=1}{\stackrel{\oplus}{\oplus} A_{j}}\right) \otimes_{F}$ $\left(\stackrel{\sim}{\otimes}_{i=1}^{r_{0}}{ }^{c} T_{i}\right) \in \mathcal{D}_{t t r}(F)$ and

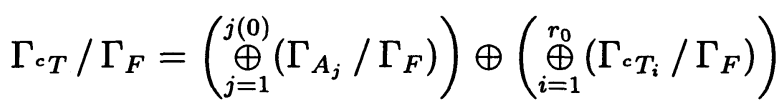

$$
\begin{aligned}
& =\left(\underset{j=0}{\stackrel{\oplus}{\oplus}(0)}\left(\Gamma_{A_{j}} / \Gamma_{F}\right)\right) \oplus[L: F]\left(\Gamma_{T^{\prime \prime}} / \Gamma_{L}\right)
\end{aligned}
$$

where $T^{\prime \prime}=\stackrel{r_{0}}{\otimes} T_{i=1}$. So $\exp \left(\Gamma_{c_{T}} / \Gamma_{F}\right)=\varepsilon \exp \left([L: F]\left(\Gamma_{T^{\prime \prime}} / \Gamma_{L}\right)\right)$ where $\varepsilon=1$ or 2. But by (4.7),

$$
\begin{aligned}
\exp \left([L: F]\left(\Gamma_{T^{\prime \prime}} / \Gamma_{L}\right)\right) & =\frac{\exp \left(\Gamma_{T^{\prime \prime}} / \Gamma_{L}\right)}{\operatorname{gcd}\left([L: F], \exp \left(\Gamma_{T^{\prime \prime}} / \Gamma_{L}\right)\right)} \\
& =\frac{s^{\prime}}{\operatorname{gcd}\left([L: F], s^{\prime}\right)} .
\end{aligned}
$$

Hence $\exp \left(\Gamma_{\mathrm{c}_{T}} / \Gamma_{F}\right)=\varepsilon s^{\prime} / \operatorname{gcd}\left([L: F], s^{\prime}\right)$.

As in (a), let $K^{\prime}$ be a totally ramified extension of $F$ with $\Gamma_{K^{\prime}}=\Gamma_{c_{T}}$. Let $n^{\prime}=\operatorname{gcd}\left([L: F], s^{\prime}\right) \cdot\left(t / s^{\prime}\right)$. Since $t(0, j) \mid 2^{e_{0}}$, we have $t(0, j) \mid n^{\prime}$. For $i>0$, we have, just as in (a), $t(i, j) \mid t^{\prime}(i, j) n^{\prime}$ if $i \leq r_{0}$ and $t(i, j) \mid n^{\prime}$ if $i>r_{0}$. These divisibility relations show $n^{\prime} \Gamma_{T} \subseteq \Gamma_{L K^{\prime}}$, hence $Z\left({ }^{c} \bar{D}\right) \subseteq \mathcal{N}(Z(\bar{D}) / \bar{F})^{1 / n^{\prime}}$, by just the same argument as for (a).

Theorem 4.6 gives us the best general relation one can expect between $Z(\bar{D})$ and $Z(\bar{c} \bar{D})$ for $D \in \mathcal{D}_{t}(L), L / F$ inertial, as the following example illustrates.

Example 4.10. Let $r_{1}, r_{2}$, and $t$ be positive integers with $t \mid[L: F]$. Let $x_{1}$, $x_{2} \in F^{*}$ such that for any prime $p$ dividing $r_{1}, r_{2}$ or $t$, we have $v\left(x_{1}\right)+p \Gamma_{F}$, $v\left(x_{2}\right)+p \Gamma_{F}$ are $\mathbb{Z}_{p}$-linearly independent in $\Gamma_{F} / p \Gamma_{F}=\Gamma_{L} / p \Gamma_{L}$. Suppose $\mu_{\ell} \subseteq F$, where $\ell=\ell \mathrm{cm}\left(r_{1}, r_{2}, t\right)$. Then if $D$ is the underlying division algebra of $\left(x_{1}, u_{1} ; L\right)_{r_{1}} \otimes_{L}\left(x_{2}, u_{2} ; L\right)_{r_{2}} \otimes\left(x_{1}, x_{2} ; L\right)_{t}$, where $u_{1}$ and $u_{2}$ are units in $L^{*}$, then by Prop. 1.4 and as $t \mid[L: F]$, in $\operatorname{Br}(F)$

$$
\begin{aligned}
{ }^{c} D \sim & \left(x_{1}, N_{L / F}\left(u_{1}\right) ; F\right)_{r_{1}} \otimes_{F} p\left(x_{1}, N_{L / F}\left(u_{2}\right) ; F\right)_{r_{2}} \\
& \otimes_{F}\left(x_{1}, x_{2}^{[L: F]} ; F\right)_{t} \\
\sim & \left(x_{1}, N_{L / F}\left(u_{1}\right) ; F\right)_{r_{1}} \otimes_{F}\left(x_{2}, N_{L / F}\left(u_{2}\right) ; F\right)_{r_{2}} .
\end{aligned}
$$


So, by Cor. 3.5, $Z(\bar{D})=\bar{L}\left(\bar{u}_{1}^{t / r_{1}}, \bar{u}_{2}^{t / r_{2}}\right)$, and $Z(\bar{c} \bar{D})=\bar{F}\left(\left(N_{\bar{L} / \bar{F}}\left(\bar{u}_{1}\right)\right)^{1 / r_{1}}\right.$, $\left.\left(N_{\bar{L} / \bar{F}}\left(\bar{u}_{2}\right)\right)^{1 / r_{2}}\right)$. Hence $Z(\bar{c} \bar{D}) \subseteq \mathcal{N}(Z(\bar{D}) / \bar{F})^{1 / t}$, where $t=\operatorname{gcd}(t,[L: F])$ as $t \mid[L: F]$.

\section{Appendix.}

In [Ta, p. 259], Tate gave a formula describing the homological corestriction using a set of representatives of the right cosets. From this, we will deduce a formula stated in Section 1 describing the homological corestriction with respect to a set of representatives of the left cosets. Presumably, this formula is known, but we have been unable to find it in the literature, except for $m \leq 2$. Here we use the notation $\overline{\sigma \rho_{i}}$ for $\rho_{\sigma_{*}(i)}$ in (1.3) above.

Let $G$ be a group and $A$ a left $G$-module. Let $H$ be a subgroup of $G$ of finite index $n$, and let $\mathcal{R}=\left\{\alpha_{1}, \ldots, \alpha_{n}\right\}$ be a set of representatives of the right cosets of $H$ in $G$. For $\tau \in G$, we write $\bar{\tau} \in R$ for the representative of $H \tau$. Given $\alpha_{i} \in \mathcal{R}$ and $\sigma \in G$, there are uniquely determined elements $\varepsilon\left(\alpha_{i}, \sigma\right) \in H$ and $\overline{\alpha_{i} \sigma} \in \mathcal{R}$ such that

$$
\alpha_{i} \sigma=\varepsilon\left(\alpha_{i}, \sigma\right) \overline{\alpha_{i} \sigma} .
$$

Then we have, for any $\sigma, \tau \in G$ and $\alpha_{i} \in \mathcal{R}$,

$$
\varepsilon\left(\alpha_{i}, 1\right)=1, \quad \text { and } \quad \varepsilon\left(\alpha_{i}, \sigma \tau\right)=\varepsilon\left(\alpha_{i}, \sigma\right) \varepsilon\left(\overline{\alpha_{i} \sigma}, \tau\right) .
$$

By [Ta, p. 259], the homological corestriction from $H^{m}(H, A)$ to $H^{m}(G, A)$ $(m \geq 1)$ can be described as follows: It is the homomorphism $\operatorname{cor}_{H}^{G}$ of the cohomology groups induced by the cochain transformation of $C^{m}(H, A)$ into $C^{m}(G, A)$, for a cochain $f \in C^{m}(H, A)$, given by

$$
\begin{aligned}
& \operatorname{cor}_{H}^{G}(f)\left(\sigma_{1}, \ldots, \sigma_{m}\right)= \\
& =\sum_{i=1}^{n} \alpha_{i}^{-1}\left[f \left(\varepsilon\left(\alpha_{i}, \sigma_{1}\right), \varepsilon\left(\overline{\alpha_{i} \sigma_{1}}, \sigma_{2}\right), \ldots, \varepsilon\left(\overline{\alpha_{i} \sigma_{1} \cdots \sigma_{j-1}}, \sigma_{j}\right)\right.\right. \\
& \left.\left.\ldots, \varepsilon\left(\overline{\alpha_{i} \sigma_{1} \cdots \sigma_{m-1}}, \sigma_{m}\right)\right)\right] .
\end{aligned}
$$

Let $\rho_{i}=\alpha_{i}^{-1}$ for $1 \leq i \leq n$, so $\mathcal{L}=\left\{\rho_{1}, \ldots, \rho_{n}\right\}$ is a set of representatives of the left cosets of $H$ in $G$. For $\tau \in G$, we write $\bar{\tau} \in \mathcal{L}$ for the representative of $\tau H$ in $\mathcal{L}$. (It will be clear from the context when $\bar{\tau}$ stands for a left coset representative and when for a right coset representative.) Given $\rho_{i} \in \mathcal{L}$ and $\sigma \in G$, there are uniquely determined elements $\overline{\sigma \rho_{i}} \in \mathcal{L}$ and $\delta\left(\sigma, \rho_{i}\right) \in H$ such that

$$
\sigma \rho_{i}=\overline{\sigma \rho_{i}} \delta\left(\sigma, \rho_{i}\right)
$$


Then, since $\overline{\sigma \tau \rho_{i}} \delta\left(\sigma \tau, \rho_{i}\right)=\overline{\sigma \bar{\tau} \rho_{i}} \delta\left(\sigma, \overline{\tau \rho_{\imath}}\right) \delta\left(\tau, \rho_{i}\right)$ for any $\sigma, \tau \in G$, and $\rho_{i} \in$ $\mathcal{L}$, we have

$$
\overline{\sigma \tau \rho_{i}}=\overline{\sigma \overline{\tau \rho_{i}}}, \quad \text { and } \quad \delta\left(\sigma \tau, \rho_{i}\right)=\delta\left(\sigma, \overline{\tau \rho_{i}}\right) \delta\left(\tau, \rho_{i}\right)
$$

Since $1=\delta\left(\sigma \sigma^{-1}, \rho_{i}\right)=\delta\left(\sigma, \overline{\sigma^{-1} \rho_{i}}\right) \delta\left(\sigma^{-1}, \rho_{i}\right)$, we have also

(A6) $\delta\left(\sigma^{-1}, \rho_{i}\right)^{-1}=\delta\left(\sigma, \overline{\sigma^{-1} \rho_{i}}\right) \quad$ for any $\quad \sigma \in G \quad$ and $\quad \rho_{i} \in \mathcal{L}$.

By taking the inverses in the both sides of (A1), we have

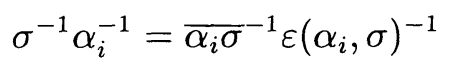

where $\overline{\alpha_{i} \sigma^{-1}} \in \mathcal{L}$ and $\varepsilon\left(\alpha_{\imath}, \sigma\right)^{-1} \in H$. But $\sigma^{-1} \alpha_{i}^{-1}=\overline{\sigma^{-1} \rho_{i}} \delta\left(\sigma^{-1}, \rho_{i}\right)$ where $\overline{\sigma^{-1} \rho_{i}} \in \mathcal{L}$ and $\delta\left(\sigma^{-1}, \rho_{i}\right) \in H$ are uniquely determined elements as in (A4). Hence we have

$$
\overline{\alpha_{i} \sigma^{-1}}=\overline{\sigma^{-1} \rho_{\imath}}
$$

and

$$
\varepsilon\left(\alpha_{i}, \sigma\right)=\delta\left(\sigma^{-1}, \rho_{i}\right)^{-1}=\delta\left(\sigma^{-1}, \alpha_{i}^{-1}\right)^{-1} .
$$

Now by using successively (A3), (A8), (A7), (A5) and (A6), the cochain transformation (A3) becomes;

$\operatorname{cor}_{H}^{G}(f)\left(\sigma_{1}, \ldots, \sigma_{m}\right)=$ 


$$
\begin{aligned}
& =\sum_{i=1}^{n} \alpha_{i}^{-1}\left[f \left(\varepsilon\left(\alpha_{i}, \sigma_{1}\right), \varepsilon\left(\overline{\alpha_{i} \sigma_{1}}, \sigma_{2}\right),\right.\right. \\
& \left.\left.\ldots, \varepsilon\left(\overline{\alpha_{i} \sigma_{1} \cdots \sigma_{j-1}}, \sigma_{j}\right), \ldots, \varepsilon\left(\overline{\alpha_{i} \sigma_{1} \cdots \sigma_{m-1}}, \sigma_{m}\right)\right)\right] \\
& =\sum_{i=1}^{n} \alpha_{i}^{-1}\left[f \left(\delta\left(\sigma_{1}^{-1}, \alpha_{i}^{-1}\right)^{-1}, \delta\left(\sigma_{2}^{-1}, \overline{\alpha_{i} \sigma_{1}}{ }^{-1}\right)^{-1}\right.\right. \text {, } \\
& \ldots \delta\left(\sigma_{j}^{-1},{\overline{\alpha_{i} \sigma_{1} \cdots \sigma_{j-1}}}^{-1}\right)^{-1} \text {, } \\
& \left.\left.\ldots, \delta\left(\sigma_{m}^{-1},{\overline{\alpha_{i} \sigma_{1} \cdots \sigma_{m-1}}}^{-1}\right)^{-1}\right)\right] \\
& =\sum_{i=1}^{n} \rho_{i}\left[f \left(\delta\left(\sigma_{1}^{-1}, \rho_{i}\right)^{-1}, \delta\left(\sigma_{2}^{-1}, \overline{\sigma_{1}^{-1} \rho_{i}}\right)^{-1},\right.\right. \\
& \cdots, \delta\left(\sigma_{j}^{-1},\left(\overline{\left.\sigma_{1} \cdots \sigma_{j-1}\right)^{-1} \rho_{i}}\right)^{-1}\right. \text {, } \\
& \left.\left.\ldots, \delta\left(\sigma_{m}^{-1},\left(\overline{\sigma_{1} \cdots \sigma_{m-1}}\right)^{-1} \rho_{i}\right)^{-1}\right)\right] \\
& =\sum_{i=1}^{n} \rho_{i}\left[f \left(\delta\left(\sigma_{1}, \overline{\sigma_{1}^{-1} \rho_{i}}\right), \delta\left(\sigma_{2}, \overline{\sigma_{2}^{-1} \sigma_{1}^{-1} \rho_{i}}\right)\right.\right. \text {, } \\
& \left.\cdots, \delta\left(\sigma_{j}, \overline{\sigma_{j}^{-1} \sigma_{j-1}^{-1} \cdots \sigma_{1}^{-1} \rho_{i}}\right), \ldots, \delta\left(\sigma_{m}, \overline{\sigma_{m}^{-1} \sigma_{m-1}^{-1} \cdots \sigma_{1}^{-1} \rho_{i}}\right)\right] \\
& =\sum_{i=1}^{n} \overline{\sigma_{1} \cdots \sigma_{m} \rho_{i}}\left[f \left(\delta\left(\sigma_{1}, \overline{\sigma_{2} \cdots \sigma_{m} \rho_{i}}\right), \delta\left(\sigma_{2}, \overline{\sigma_{3} \cdots \sigma_{m} \rho_{i}}\right)\right.\right. \\
& \left.\left.\ldots, \delta\left(\sigma_{j}, \overline{\sigma_{j+1} \cdots \sigma_{m} \rho_{i}}\right), \ldots, \delta\left(\sigma_{m}, \rho_{i}\right)\right)\right] \text {, }
\end{aligned}
$$

by replacing $\rho_{i}$ with $\overline{\sigma_{1} \cdots \sigma_{m} \rho_{i}}$, since $\left\{\overline{\sigma_{1} \cdots \sigma_{m} \rho_{1}}, \ldots, \overline{\sigma_{1} \cdots \sigma_{m} \rho_{n}}\right\}$ is a permutation of $\left\{\rho_{1}, \ldots, \rho_{n}\right\}$.

The homological corestriction $\operatorname{cor}_{H}^{G}$ from $H^{m}(H, A)$ to $H^{m}(G, A)(m \geq 1)$ is the homomorphism of cohomology groups induced by the cochain transformation $C^{m}(H, A) \rightarrow C^{m}(G, A)$ just computed.

\section{Acknowledgment.}

I am grateful to the referee for improving the proofs of Proposition 4.1 and 4.2 .

\section{References}

[Bo] N. Bourbaki, Algèbre Commutative, Chapitre VI, Valuations, Hermann, Paris, 1961.

[B] K. Brown, Cohomology of Groups, Springer-Verlag, New York, 1982.

[CF] J.W.S. Cassels and A. Frölich, Eds., Algebraic Number Theory, Academic Press, London, 1967.

[D1] P. Draxl, Skew Fields, London Math. Soc. Lecture Note Series, Vol. 81, Cambridge Univ. Press, Cambridge, 1983.

[D2] - Ostrowski's theorem for Henselian valued skew fields, J. Reine Angew. Math., 354 (1984), 213-218. 
[E] O. Endler, Valuation Theory, Springer-Verlag, New York, 1972.

[H1] Y. Hwang, The corestriction of valued division algebras over Henselian Fields, Doctoral Dissertation, University of California, San Diego, 1992.

[H2] , The corestriction of valued division algebras over Henselin Fields II, Pacific J. Math., 170 (1995), 83-103.

[JW1] B. Jacob and A. Wadsworth, A new construction of noncrossed product algebras, Trans. Amer. Math. Soc., 293 (1986), 693-721.

[JW2] — Division algebras over Henselian fields, J. Algebra, 128 (1990), 126-179.

[L] S. Lang, Algebra, Second Edition, Addison-Wesley, Menlo Park, Calibornia, 1984.

[M] A. S. Merkurjev, On the structure of the Brauer groups of fields, Izv. Akad. Nauk SSSR Ser. Mat., 49 (1985), 828-846; (English trans.) Math. USSR Izv., 27 (1986), 141-157.

[MW] P. Morandi and A. Wadsworth, Integral Dubrovin valuation rings, Trans. Amer. Math. Soc., 315, Number 2 (1989), 623-640.

[P] R. S. Pierce, Associative Algebras, Springer-Verlag, New York, 1982.

[Re] I. Reiner, Maximal Orders, Academic Press, London, 1975.

[Rb] P. Ribenboim, Equivalent forms of Hensel's lemma, Exposition. Math., 3 (1985), 3-24.

[Ri] C. Riehm, The corestriction of algebraic structures, Invent. Math., 11 (1970), 73-98.

[Ro] J.J. Rotman, An Introduction to Homological Algebra, Academic Press, New York, 1979.

[S] O.F.G. Schilling, The Theory of Valuations, Math Surveys no. 4, Amer. Math. Soc., Providence, R.I., 1950.

[Se] J.-P. Serre, Local Fields, Springer-Verlag, New York, 1979, (English trans. of Corps Locaux).

[Ta] J. Tate, Relations between $K_{2}$ and Galois Cohomology, Invent. Math., 36 (1976), 257-274.

[Ti] J.-P. Tignol, On the corestriction of central simple algebras, Math. Z., 194 (1987), 267-274.

[TW] J.-P. Tignol and A. Wadsworth, Totally ramified valuations on finite dimensional division algebras, Trans. Amer. Math. Soc., 98 (1986), 20-22.

[W1] A. Wadsworth, Extending valuations to finite dimensional division algebras, Proc. Amer. Math. Soc., 98 (1986), 20-22.

[W2] - Valuations on tensor products of symbol algebras, Azumaya Algebras, Actions and Modules (D. Haile and J. Osterburg, eds.) Contemp. Math., Vol. 124, Amer. Math. Soc., 1992, 275-289.

[We] E. Weiss, Cohomology of Groups, Academic Press, New York, 1969.

Received November 10, 1992 and revised April 8, 1993. Partially supported as a research assistant by the NSF.

Department of Mathematics

Korea UNIVERSITY

ANAM-Dong 5-1, SungBuK-Ku

SEOUL 136-701, KOREA

E-MAIL ADDRESS: YHWANG@SEMI.KOREA.AC.KR 



\title{
PACIFIC JOURNAL OF MATHEMATICS
}

Founded by E. F. Beckenbach (1906-1982) and F. Wolf (1904-1989)

\section{EDITORS}

Sun-Yung Alice Chang (Managing Editor)

University of California

Los Angeles, CA 90095-1555

pacific@math.ucla.edu

F. Michael Christ

University of California

Los Angeles, CA 90095-1555

christ@math.ucla.edu

Thomas Enright

University of California

San Diego, La Jolla, CA 92093

tenright@ucsd.edu

Nicholas Ercolani

University of Arizona

Tucson, AZ 85721

ercolani@math.arizona.edu
Robert Finn

Stanford University

Stanford, CA 94305

finn@gauss.stanford.edu

Vaughan F. R. Jones

University of California

Berkeley, CA 94720

vfr@math.berkeley.edu

Steven Kerckhoff

Stanford University

Stanford, CA 94305

spk@gauss.stanford.edu
Martin Scharlemann

University of California

Santa Barbara, CA 93106

mgscharl@math.ucsb.edu

Gang Tian

Courant Institute

New York University

New York, NY 10012-1100

tiang@taotao.cims.nyu.edu

V. S. Varadarajan

University of California

Los Angeles, CA 90095-1555

vsv@math.ucla.edu

\section{SUPPORTING INSTITUTIONS}

\section{CALIFORNIA INSTITUTE OF TECHNOLOGY \\ NEW MEXICO STATE UNIVERSITY \\ OREGON STATE UNIVERSITY \\ STANFORD UNIVERSITY \\ UNIVERSITY OF ARIZONA \\ UNIVERSITY OF BRITISH COLUMBIA \\ UNIVERSITY OF CALIFORNIA \\ UNIVERSITY OF HAWAII}

\author{
UNIVERSITY OF MONTANA \\ UNIVERSITY OF NEVADA, RENO \\ UNIVERSITY OF OREGON \\ UNIVERSITY OF SOUTHERN CALIFORNIA \\ UNIVERSITY OF UTAH \\ UNIVERSITY OF WASHINGTON \\ WASHINGTON STATE UNIVERSITY
}

The supporting Institutions listed above contribute to the cost of publication of this Journal, but they are not owners or publishers and have no responsibility for its contents or policies.

Manuscripts must be prepared in accordance with the instructions provided on the inside back cover.

The Pacific Journal of Mathematics (ISSN 0030-8730) is published monthly except for July and August. Regular subscription rate: $\$ 215.00$ a year (10 issues). Special rate: $\$ 108.00$ a year to individual members of supporting institutions.

Subscriptions, orders for back issues published within the last three years, and changes of subscribers address should be sent to Pacific Journal of Mathematics, P.O. Box 4163, Berkeley, CA 94704-0163, U.S.A. Prior back issues are obtainable from Kraus Periodicals Co., Route 100, Millwood, NY 10546.

The Pacific Journal of Mathematics at the University of California, c/o Department of Mathematics, 981 Evans Hall, Berkeley, CA 94720 (ISSN 0030-8730) is published monthly except for July and August. Second-class postage paid at Berkeley, CA 94704, and additional mailing offices. POSTMASTER: send address changes to Pacific Journal of Mathematics, P.O. Box 6143, Berkeley, CA 94704-0163.

\author{
PUBLISHED BY PACIFIC JOURNAL OF MATHEMATICS at University of California, \\ Berkeley, CA 94720, A NON-PROFIT CORPORATION \\ This publication was typeset using AMS-LATEX, \\ the American Mathematical Society's TEX macro system. \\ Copyright (C) 1995 by Pacific Journal of Mathematics
}




\section{PACIFIC JOURNAL OF MATHEMATICS}

Volume $170 \quad$ No. $1 \quad$ September 1995

Generalized generalized spin models (four-weight spin models)

EIICHI BANNAI and ETSUKO BANNAI

Fine structure of the Mackey machine for actions of abelian groups with constant Mackey obstruction

SIEGFRIED ECHTERHOFF and JONATHAN ROSENBERG

The corestriction of valued division algebras over Henselian fields. I

YOON SUNG HWANG

The corestriction of valued division algebras over Henselian fields. II

YOON SUNG HWANG

The cohomology of expansive $\mathbb{Z}^{d}$-actions by automorphisms of compact, 105 abelian groups

ANATOLE KATOK and KLAUS SCHMIDT

The Anosov theorem for exponential solvmanifolds

EDWARD KEPPELMANN and CHRISTOPHER K. MCCORD

Projections of measures on nilpotent orbits and asymptotic multiplicities 161 of $K$-types in rings of regular functions. I

DONALD RAYMOND KING

On almost-everywhere convergence of inverse spherical transforms

Christopher Meaney and Elena Prestini

Characters of supercuspidal representations of $S L(n)$

Fiona AnNe Murnaghan

The cohomology of higher-dimensional shifts of finite type

KLAUS SCHMIDT

On Gorenstein surface singularities with fundamental genus $p_{f} \geqq 2$ which satisfy some minimality conditions

TADASHI TOMARU 